\title{
INVARIANCE PRINCIPLES FOR BROWNIAN INTERSECTION LOCAL TIME AND POLYMER MEASURES
}

\author{
ANDREAS STOLL
}

\begin{abstract}
.
The goal of this article is to give a nonstandard representation of the two-dimensional VaradhanEdwards-Symanzik polymer measure by a hyperfinite Domb-Joyce model. From the standard point of view, our representation contains a new invariance principle for weakly self-avoiding or selfrepellent random walks. An important step towards this result is to give a nonstandard construction of Brownian intersection local time in $d<4$ and of its renormalization in $d=2$. Again we obtain new invariance principles similar to that in the one-dimensional case which Perkins deduced from his nonstandard approach to Brownian local time. Besides the new invariance principles, our nonstandard aproach recovers the already known existence results for the limiting objects.
\end{abstract}

\section{Introduction.}

The statistical description of polymers requires a probability measure $v$ which takes into account the 'excluded volume effect', i.e. the repulsive self-interaction of a polymer chain, which is caused by the fact that a polymer cannot loop back and cross itself. Thus Edwards [8] proposed the following polymer model: Equip the Wiener measure $\mu$ on the path space $\mathrm{C}\left([0, w], \mathrm{R}^{d}\right)$ with the formal density

$$
\frac{d v}{d \mu}(\omega)=\frac{1}{Z} \exp [-g J(\omega)]
$$

where the funtional

$$
J(\omega)=\int_{0}^{w} d s \int_{0}^{w} d t \delta(\omega(t)-\omega(s))
$$

is intended to measure the time which a Wiener path $\omega$ spends at its double points, the constant $g \in \mathrm{R}_{+}$gives the strength of the self-repulsion, $w$ is a positive real, and $Z=\int \exp (-g J) d \mu$ is the normalization contant. In dimensions $d=2$ 
and $d=3$, Varadhan [23] respectively Westwater [25], [26] could rigorously establish the polymer measure $v$ as weak limit of polymer measures $v_{n}$ where the $\delta$-function is replaced by a continuous approximation $f_{n}$. Then the functionals $J_{n}$ tend to infinity, but in dimension $d=2$, the $L^{2}$-limit of $J_{n}-E\left[J_{n}\right]$ still exists and can be used for constructing the appropriate density $d v / d \mu$ (see Varadhan [23]), whereas in dimension $d=3$ the Westwater measure $v$ is orthogonal to the Wiener measure $\mu$ (see Westwater [26]).

By nonstandard analysis, we can give a precise meaning to Edwards' heuristic approch. Choose a hyperfinite time line $T=\left\{\eta \Delta t: \eta \in{ }^{*} \mathrm{~N}_{0}\right)$ with infinitesimal spacing $\Delta t>0$ and a hyperfinite lattice $\Gamma=\left(\eta_{1} \Delta x, \ldots, \eta_{d} \Delta x\right): \eta_{t} \in * Z$ $(l=1, \ldots, \mathrm{d})\}$ with spacing $\Delta x=\sqrt{\Delta t}$. The Brownian motion is represented by a hyperfinite random walk on $\Gamma$, as it was first done by Anderson [2]. Therefore we choose an internal probability space $(\Omega, \underline{A}, \underline{P})$ on which there exists a $*$ independent internal sequence $\left(\xi_{s}\right)_{s \in T}$ of random vectors $\xi_{s}: \Omega \rightarrow^{*} Z^{d}$ with identical distribution $\underline{P} \circ \xi_{s}^{-1}={ }^{*} Q$. Define the internal process $\beta: T \times \Omega \rightarrow \Gamma$ by

$$
\beta(t, \omega)=\sum_{s=0}^{t-\Delta t} \Delta x \xi_{s}(\omega)
$$

where $\sum_{s}^{\prime}$ means $\sum_{s \in T}$; in particular we have $\beta(0, \omega)=0$. Let $(\Omega, A, P)$ be the Loeb space induced by $(\Omega, \underline{A}, \underline{P})$, i.e. $A=L(\underline{A}), P=L(\underline{P})$. We impose the following restrictions on $Q$ :

$(Q 0) Q$ is aperiodic in the sense that $Z^{d}$ is generated (as a group) by

$$
\sum(Q):=\left\{x \in Z^{d}: Q\{x\}>0\right\} .
$$

$(Q 1)$ There exists a positive real $c_{1}$ such that $Q\left\{x \in Z^{d}:|x| \geqq c_{1}\right\}=0$, i.e. $Q$ charges only a finite number of points.

$$
E(Q):=\sum\left[x Q\{x\} \mid x \in Z^{d}\right]=0 .
$$

Then it is well known that the hyperfinite random walk $\beta$ has a projection $W:\left[0, \infty\left[x \Omega \rightarrow \mathrm{R}^{d}\right.\right.$ which is a d-dimensional Brownian motion on the Loeb space $(\Omega, A, P)$ with covariance matrix

$$
\begin{gathered}
\operatorname{Cov}\left(W_{1}\right)_{j, k}=\operatorname{Cov}(Q)_{j, k}=\sum\left[x_{j} x_{k} Q\{x\} \mid x \in Z^{d}\right], \\
\text { i.e. }{ }^{\circ} \beta(t, \omega)=W\left({ }^{\circ} t, \omega\right) \quad(t \in \operatorname{ns}(T))
\end{gathered}
$$

for $P$-a.a. $\omega \in \Omega$, where ${ }^{\circ} t=\operatorname{st}(t)$ denotes the standard part of $t$ and ns $(t)$ is the set of nearstandard points in $T$.

Furthermore, in order to interprete formula (2), we need a discrete version of the $\delta$-function. If we choose 


$$
\delta(x):=\left\{\begin{array}{ll}
(\Delta x)^{-d}, & \text { if } x=0 \\
0, & \text { otherwise }
\end{array} \quad(x \in \Gamma),\right.
$$

the functional $J$ turns into an expression, which simply counts the number of double points of a given path, up to an infinitesimal constant $(\Delta t)^{2-d / 2}$. Thus, according to (1), we define a new internal probability measure $v$ on $(\Omega, \underline{A})$ by the internal density

$$
\frac{d v}{d \underline{P}}(\omega)=\frac{1}{\underline{Z}} \exp \left(-\sum\left[G \mid s, t \in T_{w} ; \beta(t, \omega)=\beta(s, \omega)\right]\right),
$$

where $G \in *^{*} R_{+}$is an internal coupling constant such that ${ }^{\circ}\left[G /(\Delta t)^{2-d / 2}\right]=g, \underline{Z}$ is the internal normalization constant, and $T_{w}=\{t \in T: t \leqq w\}$. Then, under the probability distribution $v$, the internal process $\beta$ is a hyperfinite self-repellent random walk, i.e. the probability of a path $\beta(\cdot, \omega)$ decreases with the number of its self-intersections. If $\beta$ is a simple random walk, i.e. $Q\{x\}=1 /(2 d)$ for $x \in\left\{+e_{1},-e_{1}, \ldots,+e_{d},-e_{d}\right\}$, where $e_{1}, \ldots, e_{d}$ is the standard basis of $Z^{d}$, then $(\beta, v)$ is nothing else than a hyperfinite Domb-Joyce model (see [6]).

Our main theorem states that in dimension $d=2$ the hyperfinite selfrepellent random walk $(\beta, v)$ has a projection whose distribution is Varadhan's polymer measure $v$, i.e.

$$
L(v) \circ \bar{W}^{-1}=v,
$$

where $L(v)$ is the Loeb measure on $(\Omega, A)$ induced by $v$ and $W: \Omega \rightarrow C\left([0, w), \mathrm{R}^{2}\right)$ is deduced from the Brownian motion $W$ by $\bar{W}(\omega):=W(\cdot, \omega)$. This nonstandard construction of the polymer measure contains more informtion than Varadhan's standard results in [23]. In the same manner as Anderson's nonstandard representation of Brownian motion implies Donsker's invariance principle, we obtain as a corollary that suitably scaled self-repellent random walks converge in distribution to Varadhan's polymer measure.

The crucial point in order to obtain (9) is to give a nonstandard representation of the intersection local time

$$
l(B ; x, \omega)=\int_{0}^{w} d s \int_{0}^{w} d t \chi_{B}(s, t) \delta_{x}(W(t, \omega)-W(s, \omega))
$$

of Brownian motion, where $\chi_{B}$ denotes the indicator function of a Borel set $B$ and $\delta_{x}(\mathrm{y})=\delta(\mathrm{x}-\mathrm{y})$. It will be convenient to have local times with paths in the Banach space

$$
C_{0}\left(\mathrm{R}^{d}, \mathrm{R}\right)=\left\{f: \mathrm{R}^{d} \rightarrow \mathrm{R}: f \text { continuous, bounded, and } f(x) \rightarrow 0 \text { as }|x| \rightarrow \infty\right\},
$$
equipped with the sup-norm.

In general, if $\lambda$ is any finite measure on any compact, separable time set $D$, we call a $C_{0}$-process $l: \mathrm{R}^{d} \times \Omega \rightarrow \mathrm{R}_{+}$(i.e. with paths in $C_{0}\left(\mathrm{R}^{d}, \mathrm{R}\right)$ ) the $C_{0}$-local 
time (respectively the renormalized $C_{0}$-local time) of a continuous process $Y: D \times \Omega \rightarrow \mathrm{R}^{d}$ with respect to $\lambda$, iff for $P$-a.a. $\omega \in \Omega$ we have:

$$
\int l(x, \omega) f(x) d x=\int f(Y(t, \omega)) \lambda(d t)
$$

respectively

$$
\int l(x, \omega) f(x) d x=\int\left[f(Y(t, \omega))-E f\left(Y_{t}\right)\right] \lambda(d t)
$$

e.g. for all continuous functions $f: \mathrm{R}^{d} \rightarrow \mathrm{R}$ with compact support, where $d x$ denotes the Lebesgue measure on $\mathrm{R}^{d}$. Note that the (renormalized) $C_{0}$-local time $l$ of $Y$ is unique up to indistinguishability, if it exists at all, and the existence and distribution of $l$ only depends on the distribution of $Y$. Moreover, every $h \in C_{0}\left(\mathrm{R}^{d}, \mathrm{R}\right)$ induces a linear map $\Psi \rightarrow \mathrm{R}, m \mapsto \int h d m$ on $\Psi:=\{m: m$ measure on $\mathrm{R}^{d}$ with $\left.m\left(\mathrm{R}^{d}\right) \leqq 1\right\}$ which is continuous with respect to the topology of vague convergence on $\Psi$. Therefore, if $Y$ has e.g. a $C_{0}$-local time $l$, then the random variable

$$
\int \frac{d m}{d x}(Y(t, \omega)) \lambda(d t):=\int l(x, \omega) d m(x)
$$

makes sense for every $m \in \Psi$. In particular, if $\frac{d m}{d x}=\delta_{x}$, i.e. $m$ is the Dirac measure at $x$, then

$$
l(x, \omega)=\int \delta_{x}(Y(t, \omega)) \lambda(d t) .
$$

In our case, we have $D=[0, w]^{2} \cap B, \lambda$ is the Lebesgue measure on $D$, and $Y((s, t), \omega)=W_{t}-W_{s}$. Therefore $l$ is then called intersection local time of $W$. Recently, various proofs (see e.g. Geman and Horowitz [10], Rosen [14]-[17], Yor [27], [28], Le Gall [11], Weinryb [24], Dynkin [7], Shieh [18], have been found for the following two facts:

(i) If $d<4$ and $W_{t}, W_{s}$ are independent Brownian motions, i.e. $B=[\underline{a}, \bar{a}] \times[\underline{b}, \bar{b}]$ with $0 \leqq \underline{a} \leqq \bar{a} \leqq \underline{b} \leqq \bar{b} \leqq w$, then $Y$ has a $C_{0}$-local time.

(ii) If $d=2$, then $Y$ has a renormalized $C_{0}$-local time.

However, by proving that these local times can be obtained by taking the projection (i.e. pathwise standard part) of the obvious nonstandard objects corresponding to formula (10) (as used in (8)), we show even more, namely as 
classical corollaries we obtain invariance principles for these local times. In the one-dimensional case, an invariance principle of this type was obtained for the local time of Brownian motion independently by Borodin [3] and Perkins [13], the latter using nonstandard analysis. Such a nonstandard proof mainly consists in showing that the internal local time is $S$-continuous. To this end, we shall use a nonstandard version of Kolmogorov's continuity theorem. In order to obtain the required estimates for the moments, the key idea is to use a discrete version of the Fourier inversion formula, which is based on a simple algebraic fact about the sum of unit roots.

The main estimates are contained in Section 1, where we finally arrive at the nonstandard representation of the intersection local time of independent Brownian motions in dimension in $d<4$. Then, in Section 2, it is comparably easy to derive analogous resuls for the renormalized intersection local time in dimension $d=2$ by splitting the square $[0, w]^{2}$ in Westwater's manner (see [25]). Finally, in Section 3, we only need an integrability argument in order to show that (8) actually leads to (9). We shall solve this problem by using a nonstandard version of Nelson's trick (see [12]).

For a survey on polymer models, we refer the reader to Freed [9] and Domb [5]. As an introduction to the nonstandard techniques in probability theory, we recommend the books by Albeverio et. al. [1] and Stroyan and Bayod [22]. More references can be found in the survey article by Cutland [4]. Nevertheless it is hoped that this presentation is accessible, at least on an intuitive level, to a reader with little or no knowledge of nonstandard analysis. Instead of hyperfinite models one may think of very fine discrete models and derive the same estimates for them. In our opinion, nonstandard analysis helps to understand the limiting procedures.

\section{The Intersection Local Time for Independent Brownian Motions.}

In this section, we work in dimension $d<4$. We fix an internal $\Theta: T \times T \rightarrow$ $*[0,1]$ which will be used as 'time weight'. Assuming the same setting as in the Introduction, we pick $\underline{a}, \bar{a}, \underline{b}, \bar{b} \in T$ such that $0 \leqq \underline{a} \leqq \bar{a} \leqq \underline{b} \leqq \bar{b} \leqq w$ and define for $x \in \Gamma$ and $\omega \in \Omega$ :

$$
\begin{gathered}
\rho(x, \omega):=\rho_{\Theta}(\underline{a}, \bar{a}, \underline{b}, \bar{b} ; x, \omega):= \\
\sum_{s=\underline{a}}^{a} \Delta t \sum_{t=\underline{b}}^{b} \Delta t \Theta(s, t)(\Delta x)^{-d} \chi_{\{\beta(t, \omega)-\beta(s, \omega)=x\}} .
\end{gathered}
$$

Note that the internal process $\rho: \Gamma \times \Omega \rightarrow{ }^{*} \mathrm{R}_{+}$simply counts (up to a constant) how often the difference $\beta(t, \omega)-\beta(s, \omega)$ has the value $x$. Our goal is to show that $\rho$ is $S$-continuous, i.e. that the paths of $\rho$ are infinitesimally close (in the 
sup-norm) to continuous paths. Then we can easily identify the resulting standard $C_{0}$-process as the intersection local time of $W$. We want to apply the nonstandard version of Kolmogorov's continuity theorem (see e.g. Albeverio et. al. [1]), and therefore we need estimates on the moments $\underline{E}\left(\rho_{x}-\rho_{y}\right)^{k}$. Our main technical trick is to use a discrete version of the Fourier inversion formula. This requires the following 'tuning parameters':

1.1. Notation. (i) Let $\kappa$ be the smallest even *integer such that $\kappa \Delta t / 2>1 \vee c_{1} w$, where $c_{1}$ is the constant in condition $(Q 1)$. Obviously $\kappa$ is infinite.

(ii) Let $\gamma=2 \pi /(\kappa \Delta t)$. Note that

$$
{ }^{\circ} \gamma=\pi /\left(1 \vee c_{1} w\right)>\gamma .
$$

(iii) For $k \in \mathrm{N}$, put

$$
\Gamma_{k}:=\left\{\left(n_{1} \Delta x, \ldots, n_{d} \Delta x\right):-k \kappa / 2 \leqq \eta_{l}<k \kappa / 2, \eta_{l} \in * Z \quad(l=1, \ldots, d)\right\} .
$$

Note that $|\beta(t, \omega)| \leqq(t / \Delta t) c_{1} \Delta x<\kappa \Delta x / 2$, i.e.

$\beta(t, \omega) \in \Gamma_{1}$ for all $\omega \in \Omega, \mathrm{t} \in T_{w}$.

1.2. Proposition. Let $\sigma: \Gamma_{1} \rightarrow{ }^{*} \mathrm{C}$ be internal. Define $\hat{\sigma}: \Gamma_{1} \rightarrow{ }^{*} \mathrm{C}$ by

$$
\hat{\sigma}(y)=\sum_{x \in \Gamma_{1}}(\Delta x)^{d} \sigma(x) \exp (\gamma i x \cdot y) \quad\left(y \in \Gamma_{1}\right)
$$

where $x \cdot y$ denotes the scalar product. Then

$$
\sigma(z)=\left(\frac{\gamma}{2 \pi}\right)^{d} \sum_{y \in \Gamma_{1}}(\Delta x)^{d} \hat{\sigma}(y) \exp (-\gamma i y \cdot z)
$$

for all $z \in \Gamma_{1}$.

Proof. Inserting the definition of $\hat{\sigma}$, we obtain:

$$
\begin{gathered}
\left(\frac{\gamma}{2 \pi}\right)^{d} \sum_{y \in \Gamma_{1}}(\Delta x)^{d} \hat{\sigma}(y) \exp (-\gamma i y \cdot z)= \\
\left(\frac{\gamma \Delta t}{2 \pi}\right)^{d} \sum_{x \in \Gamma_{1}} \sigma(x)\left(\sum_{y \in \Gamma_{1}} \exp [\gamma i y \cdot(x-z)]\right) .
\end{gathered}
$$

So it is sufficient to show that

$$
\tau(x, z):=\sum_{y \in \Gamma_{1}} \exp [\gamma i y \cdot(x-z)]=\left\{\begin{array}{cl}
\left(\frac{2 \pi}{\gamma \Delta t}\right)^{d}=\kappa^{d}, & \text { if } x=z \\
0 & \text {, otherwise }
\end{array}\right.
$$


for all $x, z \in \Gamma_{1}$. Since

$$
\begin{aligned}
\tau(x, z) & =\sum_{\eta_{1}=-\kappa / 2}^{-1+\kappa / 2} \ldots \sum_{\eta_{d}=-\kappa / 2}^{-1+\kappa / 2} \exp \left[\sum_{l=1}^{d} \gamma i \eta_{l} \Delta x\left(x_{l}-z_{l}\right)\right] \\
& =\prod_{l=1}^{d} \sum_{\eta_{l}=-\kappa / 2}^{-1+\kappa / 2} \exp \left[\gamma i \eta_{l} \Delta x\left(x_{l}-z_{l}\right)\right],
\end{aligned}
$$

we only have to consider the case $d=1$. Fix arbitrary $x, z \in \Gamma_{1}$. Then $x-z=\zeta \Delta x$ with $\zeta \in * Z$ such that $-(\kappa-1) \leqq \zeta \leqq(\kappa-1)$. Hence $\kappa \mid \zeta$, iff $\zeta=0$, i.e. $x=z$. This implies

$$
\begin{aligned}
\tau(x, z) & =\sum_{\eta=-\kappa / 2}^{-1+\kappa 2} \exp [\gamma \operatorname{i\eta } \xi \Delta t]=\sum_{\eta=-\kappa / 2}^{-1+\kappa / 2} \exp \left[2 \pi i \frac{\eta \zeta}{\kappa}\right] \\
& =\left\{\begin{array}{l}
\kappa, \text { if } \kappa \mid \zeta, \text { i.e. } x=z \\
0, \text { otherwise }
\end{array}\right.
\end{aligned}
$$

By Proposition 1.2, we have

$$
\rho(z, \omega)=\left(\frac{\gamma}{2 \pi}\right)^{d} \sum_{y \in \Gamma_{1}}(\Delta x)^{d} \hat{\rho}(y, \omega) \exp (-\gamma i y \cdot z) \quad\left(z \in \Gamma_{1}, \omega \in \Omega\right)
$$

with

$$
\begin{aligned}
\hat{\rho}(y, \omega) & =\sum_{x \in \Gamma_{1}}(\Delta x)^{d} \rho(x, \omega) \exp (\gamma i x \cdot y) \\
& =\sum_{s=\underline{a}}^{\bar{a}} \Delta t \sum_{t=\underline{b}}^{\bar{b}} \Delta t \Theta(s, t) \exp (\gamma i[\beta(t, \omega)-\beta(s, \omega)] \cdot y)
\end{aligned}
$$

by (14). Therefore, we get for every $k \in \mathrm{N}$ and $\mathrm{x}, \mathrm{y} \in \Gamma_{1}$ :

$$
\underline{E}\left(\rho_{x}-\rho_{y}\right)^{k}=\left(\frac{\gamma}{2 \pi}\right)^{d k} \sum_{\underline{u} \in \Gamma}(\Delta x)^{d k} q(\underset{\sim}{u}) H(\underset{\sim}{u}),
$$

where $\underset{\sim}{u}=\left(u_{1}, \ldots, u_{k}\right), \underset{\sim}{\Gamma}=\left(\Gamma_{1}\right)^{k}$,

$$
\begin{aligned}
q(\underset{\sim}{u}) & =\prod_{j=1}^{k}\left[\exp \left(-\gamma i u_{j} \cdot x\right)-\exp \left(-\gamma i u_{j} \cdot y\right)\right], \\
H(u) & =\sum_{\underline{s}=\underline{a}}^{\bar{a}} \Delta t \sum_{t=\underline{b}}^{\bar{b}}(\Delta t)^{2 k}\left[\prod_{j=1}^{k} \Theta\left(s_{j}, t_{j}\right)\right] \tau(\underset{\sim}{s}, \underset{\sim}{t} ; \underset{\sim}{u}),
\end{aligned}
$$

and

$$
\tau\left(\underline{\sim}_{\sim}, \underset{\sim}{\sim} \underset{\sim}{u}\right)=\underline{E}\left[\exp \left(\gamma i \sum_{j=1}^{k}\left[\beta\left(t_{j}\right)-\beta\left(s_{j}\right)\right] \cdot u_{j}\right)\right]
$$


Now we can do some obvious manipulations, which lead to (18)-(21). Since $\beta$ has *independent increments, we have

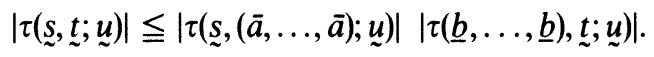

Hence

$$
|H(\underset{\sim}{u})| \leqq U(\underset{\sim}{u}) \cdot V(\underset{\sim}{u})
$$

with

$$
U(\underline{u})=\sum_{\underline{s}=\underline{a}}^{\bar{a}}(\Delta t)^{k}|\tau(\underline{\sim},(\bar{a}, \ldots, \bar{a}) ; \underset{\sim}{u})|
$$

and

$$
V(\underset{\sim}{u})=\sum_{\underline{s}=\underline{b}}^{\bar{b}}(\Delta t)^{k}|\tau((\underline{b}, \ldots, \underline{b}), \underset{\sim}{t} ; \underset{\sim}{u})| .
$$

Moreover,

$$
U(\underset{\sim}{u}) \leqq \sum_{\sigma \in T} U_{\sigma}(\underset{\sim}{u}),
$$

where $T$ is the group of permutations of $\{1, \ldots, k\}$ and

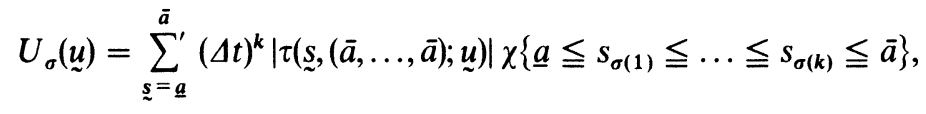

and similarly for $(\underset{\sim}{u})$. So (15) turns into

$$
\begin{aligned}
\left|\underline{E}\left(\rho_{x}-\rho_{y}\right)^{k}\right| & \left.\leqq\left(\frac{\gamma}{2 \pi}\right)^{d k} \sum_{\sigma, \sigma^{\prime} \in T} \sum_{\underset{\sim}{u} \in[}(\Delta x)^{d k}|q(\underset{\sim}{u})| U_{\sigma}(\underset{\sim}{u}) V_{\sigma^{\prime}} \underset{\sim}{u}\right) \\
& \leqq\left(\frac{\gamma}{2 \pi}\right)^{d k} \sum_{\sigma, \sigma^{\prime} \in T} \sqrt{M_{\sigma} N_{\sigma^{\prime}}}
\end{aligned}
$$

by Hölder's inequality, with

$$
M_{\sigma}=\sum_{\underline{u} \in \Gamma}(\Delta x)^{d k}|q(\underset{\sim}{u})|\left[U_{\sigma}(\underset{\sim}{u})\right]^{2}
$$

and

$$
N_{\sigma}=\sum_{\underline{u} \in \Gamma}(\Delta x)^{d k}|q(\underset{\sim}{u})|\left[V_{\sigma}(\underset{\sim}{u})\right]^{2} .
$$

Fix $\sigma \in T$. Then (16) implies

$$
U_{\sigma}(\underline{u})=\sum_{\underline{s}=\underline{a}}^{\bar{a}}(\Delta t)^{k}\left|\tau\left(s_{\sim},(\bar{a}, \ldots, \bar{a}) ; \sigma^{-1} \underline{\sim}\right)\right| \chi\left\{\underline{a} \leqq s_{1} \leqq \ldots \leqq s_{k} \leqq \bar{a}\right\}
$$


with $\sigma \underset{\sim}{u}=\left(u_{\sigma(1)}, \ldots, u_{\sigma(k)}\right)$. Next we want to rewrite $\tau(\operatorname{see}(15)$ f.):

$$
\begin{aligned}
\sum_{j=1}^{k}\left[\beta(\underline{a})-\beta\left(s_{j}\right)\right] \cdot u_{\sigma(j)} & =\sum_{j=1}^{k} \sum_{l=j}^{k}\left[\beta\left(s_{l+1}\right)-\beta\left(s_{l}\right)\right] \cdot u_{\sigma(j)} \\
& =\sum_{l=1}^{k}\left(\sum_{j=1}^{l} u_{\sigma(j)}\right) \cdot\left[\beta\left(s_{t+1}\right)-\beta\left(s_{l}\right)\right]
\end{aligned}
$$

with $s_{k+1}:=\bar{a}$. Thus we introduce the new variables $v_{l}=\sum_{j=1}^{l} u_{\sigma(j)} \in \Gamma_{l}$, i.e. $u_{\sigma(l)}=$ $v_{l-1}(l=1, \ldots, \mathrm{k})$ with $v_{0}: \equiv 0$.

Since $\beta$ has *independent identically distributed increments, we have

$$
\tau\left(s_{\sim},(\bar{a}, \ldots, \bar{a}) ; \sigma \underline{\sim}\right)=\prod_{j=1}^{k} \underline{E}\left[\exp \left(\gamma i \beta\left(s_{j+1}-s_{j}\right) \cdot v_{j}\right)\right],
$$

and therefore

$$
\begin{aligned}
U_{\sigma-1}(\underset{\sim}{u}) \leqq & \prod_{j=1}^{k}\left[\sum_{t=0}^{\bar{a}-\underline{a}} \Delta t\left|\underline{E}\left[\exp \left(\gamma i \beta_{t} \cdot v_{j}\right)\right]\right|\right] \\
& =: \sqrt{U(\bar{a}-\underline{a} ; \underset{\sim}{v})} .
\end{aligned}
$$

Consequently, the term $M_{\sigma}$ in (17) can be estimated as follows:

$$
M_{\sigma^{-1}} \leqq \sum_{\underline{v} \in \underline{\Gamma}}(\Delta x)^{d k} p(\underline{v}) U(\bar{a}-\underline{a} ; \underline{v})=: M(\bar{a}-\underline{a})
$$

with $\underline{\Gamma}=\Gamma_{1} \times \ldots \times \Gamma_{k}$ and

$$
p(v)=\prod_{j=1}^{k}\left|\exp \left[-\gamma i\left(v_{j}-v_{j-1}\right) \cdot x\right]-\exp \left[-\gamma i\left(v_{j}-v_{j-1}\right) \cdot y\right]\right| .
$$

Note that $M(\bar{a}-\underline{a})$ does not depend on the permutation $\sigma$. Similarly: $N_{\sigma} \leqq M(\bar{b}-\underline{b})$. Therefore (17) implies

$$
\left|\underline{E}\left(\rho_{x}-\rho_{y}\right)^{k}\right| \leqq\left(\frac{\gamma}{2 \pi}\right)^{d k}(k !)^{2} \sqrt{M(\bar{a}-\underline{a}) M(\bar{b}-\underline{b})} .
$$

By analogy, we have

$$
\left|\underline{E}\left(\rho_{x}\right)^{k}\right| \leqq\left(\frac{\gamma}{2 \pi}\right)^{d k}(k !)^{2} \sqrt{N(\bar{a}-\underline{a}) N(\bar{b}-\underline{b})}
$$

for all $x \in \Gamma_{1}$, with

$$
N(a)=\sum_{v \in \Gamma}(\Delta x)^{d k} U(a ; \underset{v}{v})
$$


Now a difficulty arises by the fact that our random walk $\beta$ in general is not strongly aperiodic in the sense of Spitzer [19], e.g. the simple random walk can reach 'even' lattice points only in an even number of steps. But the time weight $\Theta(s, t)$ may be chosen to be zero, if the difference $s-t$ is odd, so that $\rho_{x}$ is zero for all odd $x$. Consequently, the paths of $\rho$ are oscillating on $\Gamma$, i.e. $\rho$ cannot be $S$-continuous. However, the right hand side of (20) does not depend on $\Theta$, so it cannot give the estimate required by Kolmogorov's continuity theorem. Therefore, we have to divide $\Gamma$ into sublattices depending on the properties of the distribution $Q$ which generates the random walk $\beta$. For $n \in \mathrm{N}_{0}$ and $x, y \in Z^{d}$, we write $x \underset{Q}{\stackrel{n}{\longrightarrow}} y$, iff there exist $x_{1}, \ldots, x_{n} \in \Sigma(Q)$ (see (4)) such that $y=x+\sum_{l=1}^{n} x_{l}$. Let

$$
R_{+}(Q)=\left\{x \in Z^{d}: 0 \stackrel{n}{Q} x \text { for some } n \in N_{0}\right\} .
$$

Clearly, $R_{+}(Q)$ is a semigroup and $Z^{d}$ is the group generated by $R_{+}(Q)$ according to our aperiodicity assumption. In fact, by our assumptions on $Q$, we actually have $R_{+}(Q)=Z^{d}$. This case is not covered by P2.5 of Spitzer [19]. The elementary but somewhat tedious proof proceeds as follows: First modify the proof of P7.1 in [19] to find a basis $x_{1}, \ldots, x_{d}$ of $Z^{d}$ in $R_{+}(Q)$; then exploit the fact that $E(Q)=0$ in order to conclude that $R_{+}(Q)=Z^{d}$. In particular, there exists an integer $n>0$ with $0 \stackrel{n}{Q} 0$. Let

$$
r:=r_{Q}:=\max \{k \in \mathrm{N}: 0 \stackrel{n}{Q} 0 \text { implies } k \mid n \quad(n \in \mathrm{N})\} .
$$

For $l=0,1, \ldots, r$ define

$H_{l}(Q)=\left\{x \in \mathrm{Z}^{d}: 0 \stackrel{n r+l}{Q} x\right.$ for some $\left.n \in \mathrm{N}_{0}\right\}$

Using $R_{+}(Q)=Z^{d}$ and the definition of $r$, one can easily show that $H:=H_{0}(Q)=$ $H_{r}(Q)$ is a subgroup of $Z^{d}$, and $Z^{d}=H_{0}(Q) \cup \ldots \cup H_{r-1}(Q)$ is a disjoint union of $r$ copies of $H$. Note that $r=r_{Q}=r_{Q_{-}}$and $H_{t}(Q)=H_{r-l}\left(Q_{-}\right)(l=0,1, \ldots, r)$, where $Q_{-}\{x\}=Q\{-x\}\left(x \in Z^{d}\right)$. Let $Q_{s}$ be the distribution of $X-Y$, where $X, Y$ are independent random vectors with distribution $Q$, i.e. $Q_{s}=Q * Q_{-}$. Notice that $R_{+}\left(Q_{s}\right)=H_{0}\left(Q_{s}\right)$.

1.3. Lemma. $H=R_{+}\left(Q_{s}\right)$. 
Proof. Let $x \in R_{+}\left(Q_{s}\right)$. Then there exists $n \in \mathrm{N}$ such that $0 \underset{Q_{s}}{\stackrel{n}{\longrightarrow}} x$ and hence $0 \stackrel{n}{\longrightarrow} y \stackrel{n}{\underset{Q_{-}}{\longrightarrow}} x$ with some $y \in Z^{d}$. For some $l$, we have $y \in H_{l}(Q)$ and hence $x-y \in H_{l}\left(Q_{-}\right)=H_{r-1}(Q)$. Conversely, if $x \in H$, then there exist $n \in \mathrm{N}, y \in \mathrm{Z}^{d}$ such that $0 \underset{Q}{\stackrel{1}{\longrightarrow}} y \stackrel{n r-1}{Q} x$. Then $y \underset{Q_{-}}{\stackrel{m r+1}{\longrightarrow}} x$ for some $m \in \mathrm{N}_{0}$. For large enough $k$, say $k \geqq k_{0}$, we have $0 \stackrel{k r}{Q} 0$. Therefore $0 \stackrel{k r+1}{Q} y \frac{k r+1}{Q_{-}} x$, if $k \geqq k_{0}+m$. This implies $0 \stackrel{k r+1}{Q_{s}} x$, i.e. $x \in R_{+}\left(Q_{s}\right)$.

The further estimation of (20), (21) is based on an analysis of the Fourier transform of $Q$.

1.4. Notation. (i) Define $\phi: \mathrm{R}^{d} \rightarrow[0,1]$ by

$$
\phi(x):=\int \exp (i x \cdot y) Q_{s}(d y)=\left|\int \exp (i x \cdot y) Q(d y)\right|^{1 / 2}
$$

(ii) Let $H^{\prime}$ be the dual lattice of $H$, i.e.

$$
H^{\prime}=\left\{x \in \mathrm{R}^{d}: x \cdot y \in \mathrm{Z} \text { for all } y \in H\right\} .
$$

Then $H^{\prime}$ is a lattice in $\mathrm{R}^{d}$ with $Z^{d} \subset H^{\prime}$.

(iii) Choose a basis $e_{1}^{\prime}, \ldots, e_{d}^{\prime}$ of $H^{\prime}$. Note that $A e_{t}^{\prime}=e_{\imath}(l=1, \ldots, d)$ defines a matrix $A$ such that $H=A^{t}\left(Z^{d}\right)$ and $r=\operatorname{det} A$. Thus $H^{\prime}=A^{-1}\left(Z^{d}\right)$.

(iv) Let $C$ be the 'cube'

$$
C=\left\{x_{1} e^{\prime}+\ldots+x_{d} e_{d}^{\prime}: x_{l} \in[-\pi, \pi] \quad(l=1, \ldots, d)\right\} .
$$

Then the following follows easily from Notation 1.4, Lemma 1.3, and T7.1, P7.5 of Spitzer [19]:

1.5. Lemma. (i) For all $x, y \in \mathrm{R}^{d}$, we have:

$$
x-y \in 2 \pi H^{\prime} \text { implies } \phi(x)=\phi(y) .
$$

(ii) For all $x \in \mathrm{R}^{d}$, we have:

$$
\phi(x)=1, \text { iff } x \in 2 \pi H^{\prime} .
$$

(iii) There exists a positive real $c_{2}$ such that

$$
1-\phi(x) \geqq c_{2}|x| \text { for all } x \in C \text {. }
$$

Thus we have to adapt the variables $v_{l} \in \Gamma_{k}$ in (19) to the lattice $H^{\prime}$ :

1.6. Notation. (i) Define Int: $R^{d} \rightarrow R^{d}$, Frac: $R^{d} \rightarrow R^{d}$ by

$$
\operatorname{Int}(x):=\llbracket x_{1}+1 / 2 \rrbracket e_{1}^{\prime}+\ldots+\llbracket x_{d}+1 / 2 \rrbracket e_{d}^{\prime} \in H^{\prime},
$$




$$
\operatorname{Frac}(x):=x-\operatorname{Int}(x) \in \frac{1}{2 \pi} C,
$$

where $x=x_{1} e_{1}^{\prime}+\ldots+x_{d} e_{d}^{\prime} \in \mathrm{R}^{d}$ and $\llbracket y \rrbracket=\max \{n \in \mathrm{Z}: n \leqq y\} \quad(y \in \mathrm{R})$.

Note that for every $k \in \mathrm{N}$ :

$$
\left|\left\{\operatorname{Int}(x):\left|x \cdot e_{l}\right| \leqq k(l=1, \ldots, d), x \in \mathrm{R}^{d}\right\}\right| \leqq(2 k+1)^{d} r .
$$

Furthermore, there is a positive real $c_{3}$ such that

$$
|\operatorname{Frac}(x)| \leqq c_{3} \text { for all } x \in \mathrm{R}^{d} .
$$

(ii) For $v \in \Gamma_{k}$, define $\hat{v}, \tilde{v}$ by

$$
\hat{v}=\kappa \Delta x * \operatorname{Int}\left(\frac{v}{\kappa \Delta x}\right), \tilde{v}=\kappa \Delta x * \operatorname{Frac}\left(\frac{v}{\kappa \Delta x}\right) .
$$

Now, if $x / \Delta x \in{ }^{*} H$ and $v \in \Gamma_{k}$, then

$$
\frac{\hat{v}}{\kappa \Delta x} \epsilon^{*} H^{\prime} \text { and hence } \gamma \hat{v} \cdot x=\frac{1}{2 \pi}\left(\frac{\hat{v}}{\kappa \Delta x}\right) \cdot\left(\frac{x}{\Delta x}\right) \in * Z
$$

(recall Notation 1.1 ), i.e. $\exp [\gamma i v \cdot x]=1$. Using the elementary inequality

$$
\left|1-e^{i x \cdot y}\right| \leqq 2|x|^{\alpha}|y|^{\alpha} \quad(\alpha \in] 0,1\left[\text { and } x, y \in \mathrm{R}^{d}\right),
$$

we obtain the following estimate for (19):

1.7. Lemma. If $\alpha \in] 0,1\left[, x-y \in \Delta x^{*} H\right.$, and $\underset{\sim}{v} \in \underline{\Gamma}$, then

$$
\begin{aligned}
p(\underline{v}): & =\prod_{j=1}^{k}\left|\exp \left[-\gamma i\left(v_{j}-v_{j-1}\right) \cdot x\right]-\exp \left[-\gamma i\left(v_{j}-v_{j-1}\right) \cdot y\right]\right| \\
& \leqq \prod_{j=1}^{k}\left[2 \gamma^{\alpha}\left(\left|\tilde{v}_{j}\right|+\left|\tilde{v}_{j-1}\right|\right)^{\alpha}|x-y|^{\alpha}\right] .
\end{aligned}
$$

The estimation of $U(a ; v)$ is done in Lemmata 1.8 and 1.9.

1.8. Lemma. If $v \in \Gamma_{k}$ and $t \in T_{w}$, then

$$
\left|\underline{E}\left[\exp \left(\gamma i \beta_{t} \cdot v\right)\right]\right| \leqq \exp \left[-\frac{1}{2} c_{2} \gamma^{2}|\tilde{v}|^{2}(t-2 \Delta t)\right] \text {. }
$$

Proof. $\left|\underline{E}\left[\exp \left(\gamma i \beta_{t} \cdot v\right)\right]\right|$

$$
\begin{aligned}
& =\left|\underline{E}\left[\prod_{s=0}^{t-\Delta t} \exp \left(\gamma i \Delta x \xi_{s} \cdot v\right)\right]\right| \text {, by (3): } \\
& =\left[{ }^{*} \phi(\gamma \Delta x v)\right]^{t /(2 \Delta t)}, \text { since the } \xi_{s} \text { are } *_{\text {i.i.d. with distribution }{ }^{*} Q, \text { and }} \\
& \phi \text { is the Fourier transform of } Q_{s} ; \\
& \leqq\left[{ }^{*} \phi\left(2 \pi \frac{v}{\kappa \Delta x}\right)\right]^{[t /(2 \Delta t) \rrbracket}, \text { by Notation } 1.1(\mathrm{ii}) ;
\end{aligned}
$$




$$
\begin{aligned}
& =\left[* \phi\left(2 \pi \frac{\tilde{v}}{\kappa \Delta x}\right)\right]^{\llbracket t /(2 \Delta t) \rrbracket}, \text { by Lemma } 1.5 \text { (i), } \\
& \text { because } \frac{v}{\kappa \Delta x}-\frac{\tilde{v}}{\kappa \Delta x}=\frac{\hat{v}}{\kappa \Delta x}=\operatorname{Int}\left(\frac{v}{\kappa \Delta x}\right) \epsilon^{*} H^{\prime} ; \\
& \leqq\left[1-(2 \pi)^{2} c^{2}\left|\frac{\tilde{v}}{\kappa \Delta x}\right|^{2}\right]^{\llbracket t /(2 \Delta t) \rrbracket} \text {, by Lemma } 1.5 \mathrm{iii} ; \\
& \leqq \exp \left[-\left(\frac{2 \pi}{\kappa \Delta t}\right)^{2} c_{2}|\tilde{v}|^{2} \Delta t \llbracket t /(2 \Delta t) \rrbracket\right], \text { by } 1+x \leqq e^{x} \\
& \leqq \exp \left[-\frac{1}{2} \gamma^{2} c_{2}|\tilde{v}|^{2}(t-2 \Delta t)\right], \text { by Notation } 1.1 \text { (ii). }
\end{aligned}
$$

1.9. Lemma. If $v \in \Gamma_{k}$ and $a \in T_{w}$, then

$$
\begin{gathered}
\sum_{t=0}^{a} \Delta t\left|\underline{E}\left[\exp \left(\gamma i \beta_{t} \cdot v\right)\right]\right| \leqq \\
c_{4}\left(\frac{1}{2} c_{2} \gamma^{2}|\tilde{v}|^{2}\right)^{-1}\left(1-\exp \left[-\frac{1}{2} c_{2} \gamma^{2}|\tilde{v}|^{2}(a+\Delta t)\right]\right),
\end{gathered}
$$

where $c_{4}=\exp \left[\frac{3}{2} c_{2}\left(2 \pi c_{3}\right)^{2}\right]$, and $c_{3}$ is the constant in Notation 1.6 (i).

If $\tilde{v}=0$, the right hand side is understood to be $c_{4}(a+\Delta t)$.

ProOF. First note that Notations 1.6 and $1.1 \mathrm{imply}$

$$
|\tilde{v}|^{2} \leqq\left(\kappa \Delta x c_{3}\right)^{2}=\frac{1}{\Delta t}\left(\frac{2 \pi c_{3}}{\gamma}\right)^{2}
$$

and hence

$$
\exp \left[\frac{3}{2} c_{2} \gamma^{2}|\tilde{v}|^{2} \Delta t\right] \leqq \exp \left[\frac{3}{2} c_{2}\left(2 \pi c_{3}\right)^{2}\right]=c_{4}
$$

Then

$$
\sum_{t=0}^{a} \Delta t\left|\underline{E}\left[\exp \left(\gamma i \beta_{t} \cdot v\right)\right]\right|
$$

$\leqq \sum_{t=0}^{a} \Delta t \exp \left[-\frac{1}{2} c_{2} \gamma^{2}|\tilde{v}|^{2}(t-2 \Delta t)\right]$, by Lemma 1.8

$$
\begin{aligned}
& \leqq c_{4} \sum_{t=0}^{a} \Delta t \exp \left[-\frac{1}{2} c_{2} \gamma^{2}|\tilde{v}|^{2}(t+\Delta t)\right] \text {, by (22); } \\
& \leqq c_{4} \sum_{t=0}^{a} \int_{t}^{t+\Delta t} \exp \left[-\frac{1}{2} c_{2} \gamma^{2}|\tilde{v}|^{2} s\right]^{*} d s \\
& =c_{4} \int_{0}^{a+\Delta t} \exp \left[-\frac{1}{2} c_{2} \gamma^{2}|\tilde{v}|^{2} s\right] * d s
\end{aligned}
$$




$$
=c_{4}\left(\frac{1}{2} c_{2} \gamma^{2}|\tilde{v}|^{2}\right)^{-1}\left(1-\exp \left[-\frac{1}{2} c_{2} \gamma^{2}|\tilde{v}|^{2}(a+\Delta t)\right]\right) .
$$

Putting Lemmata 1.7, 1.8, 1.9 together, we obtain the following estimate for $M(a)$ (defined by (18), (19)), supposed that $x, y \in \Gamma_{1}, x-y \in \Delta x^{*} H, a \in T_{w}$, and $\alpha \in] 0,1[$ :

$$
\begin{gathered}
M(a) \leqq \\
\left(2 \gamma^{\alpha} c_{4}^{2}|x-y|^{\alpha}\right)^{k} \sum_{v \in \Gamma}(\Delta x)^{d k} \prod_{j=1}^{k}\left[\left(\left|\tilde{v}_{j}\right|+\left|\tilde{v}_{j-1}\right|\right)^{\alpha}\left|\tilde{v}_{j}\right|^{-4}\right. \\
\left.\left(\frac{1}{2} c_{2} \gamma^{2}\right)^{-2}\left(1-\exp \left[-\frac{1}{2} c_{2} \gamma^{2}\left|\tilde{v}_{j}\right|^{2}(a+\Delta t)\right]\right)^{2}\right] .
\end{gathered}
$$

But the terms of the sum depend only on the $\tilde{v}_{j}(j=1, \ldots, k)$, which run over the lattice

$$
\Gamma^{\prime}:=\left\{\Delta x\left(\eta_{1} e_{1}^{\prime}+\ldots+\eta_{d} e_{d}^{\prime}\right): \eta_{l} \in * \mathrm{Z},-\kappa / 2 \leqq \eta_{l}<\kappa / 2 \quad(l=1, \ldots, d)\right\},
$$

whereas $\hat{v}_{j}$ takes one of $(2 k+1)^{d} r$ possible values (see Notation 1.6).

Therefore, we get:

$$
M(a) \leqq(2 k+1)^{d k}\left(2 c_{4}^{2} r^{2}\right)^{k}|x-y|^{\alpha k}\left[\gamma^{-d}\left(2 / c_{2}\right)^{(d+\alpha) / 2}\right]^{k} S(k, \alpha, a+\Delta t)
$$

with

$$
\begin{gathered}
S(k, \alpha, a)=\sum_{v \in \Gamma^{\prime}} r^{-k}(\Delta x)^{d k} \prod_{j=1}^{k}\left[\left(\left|v_{j}\right|+\left|v_{j-1}\right|\right)^{\alpha}\left|v_{j}\right|^{-4}\right. \\
\left.\left(\sqrt{2 / c_{2}} / \gamma\right)^{4-d-\alpha}\left(1-\exp \left[-\frac{1}{2} c_{2} \gamma^{2}\left|v_{j}\right|^{2} a\right]\right)^{2}\right]
\end{gathered}
$$

and $\underset{\sim}{\Gamma^{\prime}}=\underset{i=1}{k} \Gamma^{\prime}$. Thus, from (20), (21) we obtain:

1.10. Proposition. (i) Whenever $k \in \mathrm{N}, \alpha \in] 0,1\left[\right.$, and $x, y \in \Gamma_{1}$ with $(x-y) / \Delta x \in$ ${ }^{*} H$, then

$$
\left|\underline{E}\left(\rho_{x}-\rho_{y}\right)^{k}\right| \leqq c_{5}^{k} k^{(d+2) k}|x-y|^{\alpha k}[S(k, \alpha, \bar{a}-\underline{a}+\Delta t) S(k, \alpha, \bar{b}-\underline{b}+\Delta t)]^{\frac{1}{2}}
$$

with $c_{5}=2 c_{4}^{2} r^{2}\left(1 \vee 2 / c_{2}\right)^{(d+1) / 2}$.

(ii) For all $x \in \Gamma_{1}$ and $k \in \mathrm{N}$, we have

$$
\left|\underline{E}\left(\rho_{x}\right)^{k}\right| \leqq c_{5}^{k} k^{(d+2) k}[S(k, 0, \bar{a}-\underline{a}+\Delta t) S(k, 0, \bar{b}-\underline{b}+\Delta t)]^{\frac{1}{2}} .
$$

The terms $S(k, \alpha, a)$ can be estimated as follows:

1.11. Proposition. For every

$$
\alpha \in\left\{\begin{array}{l}
{[0,1[, \text { if } d \in\{1,2\}} \\
{\left[0, \frac{1}{2}[, \text { if } d=3\right.}
\end{array}\right.
$$

there exists a positive real $c_{6}=c_{6}(d, \alpha)$ such that

$$
S(k, \alpha, a) \leqq\left[c_{6} a^{(4-d-\alpha) / 2}\right]^{k}
$$


for all finite $a \in{ }^{*} \mathrm{R}_{+}$and $k \in \mathrm{N}$.

Proof. Put $\Delta \overline{\mathrm{x}}:=\sqrt{\frac{1}{2} c_{2} \gamma^{2} a} \Delta x$ and $\bar{\Gamma}:=\Delta \bar{x}^{*} H^{\prime}$.

Then (23) scales to

$$
\begin{gathered}
S(k, \alpha, a) \leqq \\
\left\{\sum _ { v \in [ } r ^ { - k } ( \Delta \overline { x } ) ^ { d k } \prod _ { j = 1 } ^ { k } \left[\left(\left|v_{j}\right|+\left|v_{j-1}\right|\right)^{\alpha}\left|v_{j}\right|^{-4}\right.\right. \\
\left.\left.\left(1-\exp \left[-\left|v_{j}\right|^{2}\right]\right)^{2}\right]\right\} a^{(4-d-\alpha) k / 2} .
\end{gathered}
$$

Let us first assume that $\alpha \neq 0$. Then we choose $\varepsilon \in] 0,4[$ such that with $\varepsilon^{\prime}=4-\varepsilon, \alpha^{\prime}=1-\alpha$, we have $\varepsilon / \alpha-2>d$ and $\varepsilon^{\prime} / \alpha^{\prime}>d$. E.g. we can take $\varepsilon=4 \alpha+1 / 2$, if $d=3$ and $\alpha \in] 0,1 / 2[$, and $\varepsilon=3 \alpha+1$, if $d \in\{1,2\}$ and $\alpha \in] 0,1[$. Then we can apply Hölder's inequality:

$$
\begin{gathered}
a^{(d+\alpha-4) k / 2} S(k, \alpha, a) \leqq\left(\sum_{v \in \Gamma} r^{-k}(\Delta \bar{x})^{d k} \prod_{j=1}^{k}\left[\left(\left|v_{j}\right|+\mid v_{j-1}\right) F\left(v_{j}\right)^{\varepsilon / \alpha}\right]\right)^{\alpha} \\
\left(\sum_{v \in[} r^{-k}(\Delta \bar{x})^{d k} \prod_{j=1}^{k} F\left(v_{j}\right)^{\varepsilon^{\prime} / \alpha^{\prime}}\right)^{\alpha^{\prime}}=:\left[S_{1}(k, \alpha, a)\right]^{\alpha}\left[S_{2}\left(k, \alpha^{\prime}, a\right)\right]^{\alpha^{\prime}},
\end{gathered}
$$

with $F(v)=|v|^{-1}\left[1-\exp \left(-|v|^{2}\right)\right]^{\frac{1}{2}}$.

Furthermore

$$
\prod_{j=1}^{k}\left(\left|v_{j}\right|+\left|v_{j-1}\right|\right) \leqq 2^{k} \prod_{j=1}^{k}\left(\left|v_{j}\right|^{2} \vee 1\right)
$$

Therefore

$$
S_{1}(k, \alpha, a) \leqq\left[2 \sum_{v \in \Gamma} r^{-1}(\Delta \bar{x})^{d}\left(|v|^{2} \vee 1\right) F(v)^{\varepsilon / \alpha}\right]^{k}
$$

and

$$
S_{2}\left(k, \alpha^{\prime}, a\right) \leqq\left[\sum_{v \in \Gamma} r^{-1}(\Delta \bar{x})^{d} F(v)^{\varepsilon^{\prime} / \alpha^{\prime}}\right]^{k} .
$$

Note that the lattice $\bar{\Gamma}$ with weighting $\Delta \bar{x} / r$ is a hyperfinite representation of the Lebesgue measure in $R^{d}$ (cf. Stroyan and Bayod [22]. So it is certainly enough to show that the functions

$$
\bar{\Gamma} \rightarrow^{*} \mathrm{R}, v \mapsto\left(|v|^{2} \vee 1\right) F(v)^{\varepsilon / \alpha} \text { respectively } v \mapsto F(v)^{\varepsilon^{\prime} / \alpha^{\prime}}
$$

are $S$-integrable. But this is obvious, because $F$ has a finite bound and both $\varepsilon / \alpha-2$ and $\varepsilon^{\prime} / \alpha^{\prime}$ are greater than $d$.

The case $\alpha=0$ is analogous.

Fix $\imath \in\{0,1, \ldots, r\}$ and let 


$$
\Xi_{l}=\left\{(\underline{a}, \bar{a}, \underline{b}, \bar{b} ; x): x / \Delta x \in{ }^{*} H_{i} ; 0 \leqq \underline{a} \leqq \bar{a} \leqq \underline{b} \leqq \bar{b} \leqq w ; \underline{a}, \bar{a}, \underline{b}, \bar{b} \in T\right\} .
$$

Then Propositions 1.10 and 1.11 show that there exists a positive real $c_{7}=$ $c_{7}(d, k, w)$ such that

$$
\left|\underline{E}\left(\rho_{u}-\rho_{v}\right)^{k}\right| \leqq c_{7}|u-v|^{(1-d / 4) k}
$$

for all finite $u, v \in \Xi_{\text {l }}$ and $k \in N$. Choosing e.g. $k=32$, we have $(1-d / 4) k>d+4$, so that the nonstandard version of Kolmogorov's continuity theorem (see e.g. Albeverio et al. [1]) implies that $P$-almost all paths of the internal process $\rho: \Xi_{l} \times \Omega \rightarrow{ }^{*} R_{+}$are $S$-continuous.

Moreover, by (6) we have for $P$-a.a. $\omega \in \Omega$ :

$$
\rho(x, \omega)=0 \text { for all infinite } x .
$$

Consequently, $P$-a.a. paths of $\rho$ are nearstandard in $C_{0}(M, \mathrm{R})$ with

$$
M=\left\{(\underline{a}, \bar{a}, \underline{b}, \bar{b} ; x) \in \mathrm{R}^{4+d}: 0 \leqq \underline{a} \leqq \bar{a} \leqq \underline{b} \leqq \bar{b} \leqq w\right\} .
$$

Therefore $\rho\left\lceil\Xi\right.$ has a projection $l_{l}$, i.e. $l_{l}: M \times \Omega \rightarrow \mathrm{R}_{+}$is a $C_{0}$-standard process such that

$$
{ }^{\circ} \rho(u, \omega)=l_{l}\left({ }^{\circ} u, \omega\right) \quad\left(u \in \Xi_{l}\right)
$$

holds for $P$-a.a. $\omega \in \Omega$, where ${ }^{\circ} u=\infty$ for $|u|$ infinite and $l,(\infty, \omega)=0$.

For $l=1, \ldots, r$, define $\Theta_{l}: T \times T \rightarrow{ }^{*}[0,1]$ by

$$
\Theta_{\imath}(s, t):= \begin{cases}\Theta(s, t), & \text { if }(s-t) / \Delta t \in r^{*} Z+\imath \\ 0, & \text { otherwise }\end{cases}
$$

Note that $\beta(t, \omega)-\beta(s, \omega) \in \Delta x^{*} H_{l}$, iff $(s-t) / \Delta t \in r^{*} Z+l$.

Therefore $\rho_{\boldsymbol{\theta}}=\sum_{l=1}^{r} \rho_{\boldsymbol{\theta}_{l}}$ and $\rho_{\boldsymbol{\theta}} \uparrow \Xi_{l}=\rho_{\boldsymbol{\theta}_{l}} \uparrow \Xi_{l}=: \rho(l=1, \ldots, r)$.

So we cannot expect that $\rho_{\theta}$ is $S$-continuous in general. Let $\underline{\lambda}$ be the internal measure on $T \times T$ with weight $\lambda\{(s, t)\}=(\Delta t)^{2}$, and $L(\underline{\lambda})$ the induced Loeb measure. Then $\lambda=L(\underline{\lambda}) \circ \mathrm{st}^{-1}$ is the Lebesgue measure on $\mathrm{R}_{+} \times \mathrm{R}_{+}$. Obviously the measure $L\left(\Theta_{l} \underline{\lambda}\right) \circ \mathrm{st}^{-1}$ on $\mathbf{R}_{+} \times \mathbf{R}_{+}$, which is induced by the internal measure $\Theta_{l} \underline{\lambda}\{(s, t)\}=\Theta(s, t)(\Delta t)^{2}$ on $T \times T$, has a Lebesgue density $\tilde{\theta}_{l}: \mathbf{R}_{+} \times \mathbf{R}_{+} \rightarrow[0,1]$, i.e. $L(\Theta, \underline{\lambda}) \circ \mathrm{st}^{-1}=\tilde{\Theta}, \lambda$. Note that every measurable $\Theta: R_{+}^{2} \rightarrow[0,1]$ for every $l$ can be represented this way, i.e. $\theta=\tilde{\Theta}, \lambda$-a.s. with some $\Theta$.

1.12. THEOREM. For every $u=(\underline{a}, \bar{a}, \underline{b}, \bar{b}) \in \mathrm{R}^{4}$ with $0 \leqq \underline{a} \leqq \bar{a} \leqq \underline{b} \leqq \bar{b} \leqq w$, the process $Y: D \times \Omega \rightarrow R, Y(s, t ; \omega)=W(t, \omega)-W(s, \omega)$, where $D=[\underline{a}, \bar{a}] \times[\underline{b}, \bar{b}]$ and $W$ is given by (6), has a $C_{0}$-local time $\frac{1}{r} l,(u ;-,-): \mathbf{R}^{d} \times \Omega \rightarrow \mathrm{R}_{+}$with respect to the time measure $\tilde{\Theta}_{l} \lambda\left\lceil D\right.$. Moreover, $l_{l}$ can be chosen jointly continuous and is given by (24). 
Proof. Fix $\omega \in \Omega$ such that (6) and (24) hold. Pick $u=(\underline{a}, \bar{a}, \underline{b}, \bar{b}) \in T^{4}$ such that $0 \leqq \underline{a} \leqq \bar{a} \leqq \underline{b} \leqq \bar{b} \leqq w$ and put $D=\left[{ }^{\circ},{ }^{\circ} \bar{a}\right] \times\left[{ }^{\circ} \underline{b},{ }^{\circ} \bar{b}\right], B=\{(s, t) \in T \times T$ : $\underline{a} \leqq s \leqq \bar{a}, \underline{b} \leqq t \leqq \bar{b}\}$. Let $f: \mathrm{R}^{d} \rightarrow \mathrm{R}$ be an arbitrary continuous function with compact support. Then we have to check (11):

$$
\begin{gathered}
\int_{D} f(W(t, \omega)-W(s, \omega)) \tilde{\Theta}_{l}(s, t) d \underline{\hat{\lambda}}(s, t) \\
=\int \chi\left[T^{2} \cap \mathrm{st}^{-1}(D)\right](s, t) f\left(W\left({ }^{\circ} t, \omega\right)-W\left({ }^{\circ} s, \omega\right)\right) d L\left(\Theta_{l} \underline{\lambda}\right)(s, t),
\end{gathered}
$$

since $L\left(\Theta_{l} \underline{\lambda}\right) \circ$ st $^{-1}=\tilde{\Theta_{l}} \underline{\lambda}$

$$
\begin{gathered}
=\int_{B} f\left({ }^{\circ} \beta(t, \omega)-{ }^{\circ} \beta(s, \omega)\right) d L\left(\Theta_{l} \underline{\lambda}\right)(s, t), \text { by }(6) \text { and } L\left(\Theta_{l} \underline{\lambda}\right) \ll L(\underline{\lambda}) ; \\
=\int_{B} s t^{*} f(\beta(t, \omega)-\beta(s, \omega)) d L\left(\Theta_{l} \underline{\lambda}\right)(s, t), \text { by the continuity of } f ; \\
={ }^{\circ} \sum_{s=\underline{a}}^{\bar{a}} \Delta t \sum_{s=\underline{b}}^{\bar{b}} \Delta t \Theta_{l}(s, t)^{*} f(\beta(t, \omega)-\beta(s, \omega),
\end{gathered}
$$

since the internal function $(s, t) \mapsto^{*} f(\beta(t, \omega)-\beta(s, \omega))$ is $S$-integrable with respect to $\Theta_{l} \underline{\lambda}\lceil\underline{B}$

$$
\begin{gathered}
=\sum_{s=\underline{a}}^{\bar{a}} \Delta t \sum_{s=\underline{b}}^{\bar{b}} \Delta t \Theta_{l}(s, t) \sum_{x \in \Gamma} \chi\{\omega(t)-\omega(s)=x\}^{*} f(x), \\
\text { since } \omega(t)-\omega(s) \in \Gamma \text { for all }(s, t) \in T^{2} ; \\
=\sum_{\Delta x^{*} H_{l}}(\Delta x)^{d} \rho(u ; x, \omega)^{*} f(x), \text { by the definition of } \rho(\text { see }(14)) ; \\
\int_{\Delta x^{*} H_{l}} \operatorname{st}\left[\rho(u ; x, \omega)^{*} f(x)\right] d L(\Delta x)^{d}(x),
\end{gathered}
$$

by $S$-integrability (use the $S$-continuity of $\rho \mid \Xi_{l}$ );

$$
\begin{gathered}
=\int_{\Delta x^{*} \boldsymbol{H}_{l}} l\left({ }^{\circ} u ;{ }^{\circ} x, \omega\right) f\left({ }^{\circ} x\right) d L(\Delta x)^{d}(x), \text { by }(24) \text { and the continuity of } f ; \\
\left.=\frac{1}{r} \int_{\mathrm{ns}(\Gamma)} l,{ }^{\circ} u ;{ }^{\circ} u, \omega\right) f\left({ }^{\circ} x\right) d L(\Delta x)^{d}(x) \\
\left.=\frac{1}{r} \int l l_{l}{ }^{\circ} u, x, \omega\right) f(x) d x,
\end{gathered}
$$

since $L(\Delta x)^{d} \circ \mathrm{st}^{-1}$ is the Lebesgue measure on $\mathrm{R}^{d}$. 
The new point in Theorem 1.12 is that the intersection local time $l_{l}$ is given by (24). Because then a routine argument, which was employed e.g. by Anderson [2], Perkins [13], or Stoll [20], [21], shows the following:

1.13. INVARIANCE PRINCIPLE. (i) If $\tilde{\Theta}_{1}=\ldots=\tilde{\Theta}_{r}=: \frac{1}{r} \theta$, then $\rho_{\Theta}$ is $S$-continuous with projection $l=l_{1}=\ldots=l_{r}$, which is the local time of $Y$ with respect to the time measure $\Theta \lambda\lceil D$.

(ii) Let $K$ be the distribution of the random funtion

$$
\Omega \rightarrow C_{0}\left([0, w] \times M, \mathrm{R}^{d} \times \mathrm{R}\right), \omega \rightarrow(\bar{W}(\omega) ;((\omega)),
$$

where $\bar{W}(\omega):[0, w] \rightarrow \mathrm{R}^{d}, t \rightarrow W(t, \omega)$ is a $d$-dimensional Brownian motion (with covariance matrix (5)) and $\bar{l}(\omega): M \rightarrow \mathrm{R}, u \rightarrow l(u, \omega)$ is the corresponding intersection local time (as given by (24)) with respect to a measurable time density $\Theta: \mathrm{R}_{+} \times \mathrm{R}_{+} \rightarrow[0,1]$. For each $n \in \mathrm{N}$, let $\left(\Omega_{n}, A_{n}, P_{n}\right)$ be a probability space, $\Delta t_{n}$ a positive real, $\Delta x_{n}=\sqrt{\Delta t_{n}}, T_{n}=\left\{k \Delta t_{n}: k \in \mathrm{N}_{0}\right\}, T_{n, w}=[0, w] \cap T_{n}, M_{n}=$ $\left\{(\underline{a}, \bar{a}, \underline{b}, \bar{b} ; x): x \in \Delta x_{n} Z^{d} ; \underline{a}, \bar{a}, \underline{b}, \bar{b} \in T_{n} ; 0 \leqq \underline{a} \leqq \bar{a} \leqq \underline{b} \leqq \bar{b} \leqq w\right\}$, $\Theta_{n}: T_{n}^{2} \rightarrow[0,1]$ an arbitrary function, and $\left(X_{n, t} \mid t \in T_{n}\right)$ a sequence of independent random vectors on $\Omega_{n}$ with distribution $P_{n} \circ \chi_{n, t}^{-1}=Q$. Moreover, for each $\imath=1, \ldots, r$ define $\Theta_{n, l}: T_{n}^{2} \rightarrow[0,1]$ by

$\Theta_{n, l}(s, t)=\left\{\begin{array}{ll}\Theta_{n}(s, t), & \text { if }(s-t) / \Delta t_{n} \in r Z+\imath \\ 0, & \text { otherwise }\end{array}\right.$,

and let $\lambda_{n}$ be the measure on $T_{n} \times T_{n}$ with $\lambda_{n}\{(s, t)\}=\left(\Delta t_{n}\right)^{2}$. For each $n \in \mathrm{N}$, define the processes $\beta_{n}: T_{n, w} \times \Omega_{n} \rightarrow \mathrm{R}^{d}, \rho_{n}: M_{n} \times \Omega_{n} \rightarrow \mathrm{R}+$ by

$\beta_{n}(t, \omega)=\sum\left[X_{n, s}(\omega) \Delta x_{n} \mid s \in T_{n}, s<t\right]$, respectively

$\rho_{n}(\underline{a}, \bar{a}, \underline{b}, \bar{b} ; x, \omega)=\sum\left[\left(\Delta t_{n}\right)^{2} \Theta_{n}(s, t)\left(\Delta x_{n}\right)^{-d} \mid s, t \in T_{n} ;\right.$

$$
\left.\underline{a} \leqq s \leqq \bar{a}, \underline{b} \leqq t \leqq \bar{b} ; \beta_{n}(s, \omega)=\beta_{n}(t, \omega)\right],
$$

and the random functions $\bar{\beta}_{n}: \Omega_{n} \rightarrow F_{0}\left(T_{n, w}, \mathrm{R}^{d}\right)=\left\{f \uparrow T_{n, w}: f \in C_{0}\left(\mathrm{R}_{+}, \mathrm{R}^{d}\right)\right\}$, $\bar{\rho}_{n}: \Omega_{n} \rightarrow F_{0}\left(M_{n}, \mathrm{R}\right)$ by $\bar{\beta}_{n}(\omega): T_{n, w} \rightarrow \mathrm{R}^{d}, t \rightarrow \beta_{n}(t, \omega)$, respectively $\bar{\rho}_{n}(\omega): M_{n} \rightarrow \mathrm{R}$, $\mathrm{u} \mapsto \rho_{n}(u, \omega)$. For each $n \in \mathrm{N}$, let $K_{n}$ be the probability measure on $F_{0}\left(T_{n, w} \times M_{n}\right.$, $\left.\mathrm{R}^{d} \times \mathrm{R}\right)$ induced by the random function $\left(\bar{\beta}_{n}, \bar{\rho}_{n}\right)$. Suppose that $\lim \Delta t_{n}=0$ and $\Theta_{n, l} \lambda_{n} \underset{n \rightarrow \infty}{\stackrel{\text { vaguely }}{\longrightarrow}} \frac{1}{r} \theta \lambda$ (that means ${ }^{*} \Theta_{\eta, l} \cong \frac{1}{r} \theta \lambda$ for all infinite $\eta$ ) for $\imath=1, \ldots, \mathrm{r}$.

Then we have

$$
\left(\bar{\beta}_{n}, \bar{\rho}_{n}\right) \underset{n \rightarrow \infty}{\stackrel{w}{\longrightarrow}}(\bar{W}, \bar{l}),
$$


i.e. $K_{n} \circ I_{n}^{-1} \stackrel{\text { weakly }}{n \rightarrow \infty} K$ for every reasonable sequence $\left(I_{n}\right)_{n \in \mathbb{N}}$ of interpolations $I_{n}: F_{0}\left(T_{n, w} \times M_{n}, \mathrm{R}^{d} \times \mathrm{R}\right) \rightarrow C_{0}\left([0, w] \times M, \mathrm{R}^{d} \times \mathrm{R}\right)$.

'Reasonable' means that ${ }^{*} I_{\eta}$ preserves $S$-continuity for every infinite $\eta$; however it is possible to extend the classical notion of weak convergence to cases like (24) without referring to a particular interpolation procedure (see e.g. Stoll [20]).

\section{The Renormalized Intersection Local Time of Planar Brownian Motion.}

For the rest of this article we shall restrict ourselves to the case $d=2$. In this section, we shall study the 'full' internal intersection local time

$$
\begin{aligned}
\tau(x, \omega): & =\tau_{\boldsymbol{\theta}}(v ; x, \omega) \\
& =\sum\left[\Delta t \Theta(s, t) \mid s, t \in T_{v} ; \beta(t, \omega)-\beta(s, \omega)=x\right] \quad(\omega \in \Omega, x \in \Gamma),
\end{aligned}
$$

with $T_{v}=\{t \in T: t \leqq v\}, v \in \mathrm{ns}\left({ }^{*} \mathrm{R}_{+}\right)$, and $\Theta: T \times T \rightarrow{ }^{*}[0,1]$ internal. In order to get a standard part of $\tau$, it will be necessary to renormalize $\tau$ by subtracting its expectation, which is infinite in general. So, for any internal process $\sigma(x, \omega)$ the renormalized process is defined by $\dot{\sigma}(x, \omega)=\sigma(x, \omega)-\underline{E} \sigma_{x}$. For the renormalized $\tau$ we can prove similar resuls as we did for $\rho$ in Section 1. First we shall show that $\tau$ is jointly $S$-continuous by establishing estimates analogous to Propositions 1.10 and 1.11. Then the standard part of $\tau$ turns out to be the renormalized intersection local time of the planar Brownian motion $W$. We can make use of the results in Section 1, if we split the domain $T_{v} \times T_{v}$ by Westwater's manner (see [25]). To this end, we fix $v \geqq \Delta t$ (otherwise $\tau \equiv 0$ ) and put

$$
\kappa:=\kappa^{v}:=\min \left\{\eta \in{ }^{*} N_{0}: 2^{\eta} \Delta t>v\right\},
$$

i.e. $v<2^{\kappa} \Delta t \leqq 2 v \quad$ (forget Notation 1.1).

For every $\xi \in\{0,1, \ldots, \kappa\}$, we define the following domains:

2.1. Notation. (i) If $\eta \in\left\{0,1, \ldots, 2^{\xi}-1\right\}$, put

$\Delta_{\xi}(\eta)=\left\{(s, t) \in T^{2}:\right.$ there are $s_{0}, t_{0} \in T$ such that $s_{0}, t_{0}<2^{\kappa-\xi} \Delta t$

$$
\text { and } \left.s=\eta 2^{\kappa-\xi} \Delta t+s_{0}, t=\eta 2^{\kappa-\xi} \Delta t+t_{0}\right\},
$$

$\Lambda_{\xi}(\eta, 1)=\left\{(s, t) \in T^{2}\right.$ : there are $s_{0}, t_{0} \in T$ such that $s_{0}, t_{0}<2^{\kappa-\xi-1} \Delta t$

$$
\text { and } \left.s=(\eta+1 / 2) 2^{\kappa-\xi} \Delta t+s_{0}, t=\eta 2^{\kappa-\xi} \Delta t+t_{0}\right\},
$$

$\Lambda_{\xi}(\eta, 2)=\left\{(s, t) \in T^{2}\right.$ : there are $s_{0}, t_{0} \in T$ such that $s_{0}, t_{0}<2^{\kappa-\xi-1} \Delta t$

$$
\text { and } \left.s=\eta 2^{\kappa-\xi} \Delta t+s_{0}, t=(\eta+1 / 2) 2^{\kappa-\xi} \Delta t+t_{0}\right\},
$$

(ii) Let

$$
\begin{aligned}
& \Delta_{\xi}=\bigcup\left\{\Delta_{\xi}(\eta): \eta=0,1, \ldots, 2^{\xi-1}\right\} \\
& \Lambda_{\xi}=\bigcup\left\{\Lambda_{\xi}(\eta): \eta=0,1, \ldots, 2^{\xi-1}\right\}
\end{aligned}
$$


(iii) If $\Delta$ is an arbitrary internal subset of $T^{2}$, define the internal process $\tau_{\boldsymbol{\theta}}^{v}[\Delta]: \Gamma \times \Omega \rightarrow{ }^{*} \mathrm{R}_{+}$by $\tau_{\boldsymbol{\theta}}^{v}[\Delta]=\tau_{\overline{\boldsymbol{\theta}}}(v ;-,-)$ with $\bar{\Theta}=\Theta \cdot \chi_{\Delta}$. In particular, put for all $\xi \in\{0,1, \ldots, \kappa\}$ :

$$
\begin{gathered}
\sigma_{\xi}:=\tau_{\boldsymbol{\theta}}^{v}\left[\Delta_{\xi}\right], \rho_{\xi}:=\tau_{\boldsymbol{\theta}}^{v}\left[\Lambda_{\xi}\right], \text { and } \\
\tau_{\xi}:=\tau_{\boldsymbol{\theta}}^{v}[\Delta] \text { with } \Delta=\bigcup\left\{\Lambda_{\zeta}: \zeta=0,1, \ldots, \xi-1\right\} .
\end{gathered}
$$

Note that $\Delta_{0}=\left\{(s, t) \in T^{2}: s, t<2^{\kappa} \Delta t\right\}, \Delta_{\kappa}=\left\{(s, t) \in T^{2}: s=t<2^{\kappa} \Delta t\right\}$, and $\Lambda_{\kappa}=\emptyset$.

Moreover, for all $\xi \in\{0,1, \ldots, \kappa-1\}$ and $\eta \in\left\{0,1, \ldots, 2^{\xi}-1\right\}$ we have $\Delta_{\xi}(\eta)=\Lambda_{\xi}(\eta) \cup \Delta_{\xi+1}(2 \eta) \cup \Delta_{\xi+1}(2 \eta+1), \Delta_{\xi+1}=\Delta_{\xi} \backslash \Lambda_{\xi}$, and

$$
\tau=\sigma_{\xi}+\tau_{\xi}=\sigma_{\xi}+\sum_{\zeta=0}^{\xi-1} \rho_{\zeta} .
$$

In order to estimate the moments of $t$, it will be convenient to use Lemma 5 of Westwater [25]. The proof of this lemma immediately gives the following nonstandard version:

2.2. Lemma. Let $X_{l}, l \in * \mathrm{~N}$, be an internal sequence of *independent random variables. Suppose there exist positive reals $c_{8}, c_{9}$ such that $\left\|X_{l}\right\|_{k}:=\left(\underline{E}\left[X_{l}^{k}\right]\right)^{1 / k} \leqq$ $c_{8} k^{c 9}$ for all even $k \in \mathrm{N}$ and all $\iota \in{ }^{*} \mathrm{~N}$. For $\eta \in *^{*} \mathrm{~N}$, put $N_{\eta}=\frac{1}{\sqrt{\eta}} \sum_{i=1}^{\eta}\left(X_{l}-\underline{E} X_{l}\right)$.

Then $\left\|N_{\eta}\right\|_{k} \leqq 2 c_{8} k^{c_{9}+1 / 2}$ for all even $k \in \mathrm{N}$ and all $\eta \in * \mathrm{~N}$.

From Propositions 1.10, 1.11 it follows that, with $\bar{v}=2^{\kappa-\xi-1} \Delta t$ and $\bar{\Theta}(s, t)=\Theta\left(s+\eta 2^{\kappa-\xi} \Delta t, t+\eta^{2 \kappa-\xi} \Delta t\right) \cdot \chi_{T_{v}^{2}}^{2}(s, t)$, we have

$$
\begin{aligned}
\left\|\tau_{\Theta}^{v}\left[\Lambda_{\xi}(\eta, 2)\right](x,-)\right\|_{k} & =\left\|\rho_{\bar{\theta}}(0, \bar{v}-\Delta t, \bar{v}, 2 \bar{v}-\Delta t ; x,-)\right\|_{k} \\
& \leqq c_{5} k^{d+2} c_{6}\left(2^{\kappa-\xi-1} \Delta t\right)^{2-d / 2} \leqq c_{5} c^{6} k^{4} v 2^{-\xi}
\end{aligned}
$$

for all $k \in \mathrm{N}, \xi \in\{0,1, \ldots, \kappa-1\}, \eta \in\left\{0,1, \ldots, 2^{\xi}-1\right\}, x \in \Gamma_{1}$.

So we can apply Lemma 2.2 with

$$
X_{l}=\tau_{\theta}^{v}\left[\Lambda_{\xi}(\eta)\right](x,-) \cdot 2^{\xi} / v
$$

and obtain

$$
\left\|\dot{\rho}_{\xi}(x,-)\right\|_{k} \leqq 4 c_{5} c_{6} k^{9 / 2}\left(v 2^{-\xi}\right) \sqrt{2^{\xi}}
$$

and hence

$$
\left\|\dot{\tau}_{x}\right\|_{k} \leqq \sum_{\xi=0}^{\kappa-1}\left\|\dot{\rho}_{\xi}(x,-)\right\|_{k} \leqq 4 c_{5} c_{6} k^{9 / 2} v\left(1-2^{-1 / 2}\right)^{-1}
$$

for all $k \in 2 \mathrm{~N}, x \in \Gamma_{1}$. With a similar procedure for $\dot{\tau}_{x}-\dot{\tau}_{y}$ we obtain: 
2.3. Proposition. (i) Whenever $k \in 2 \mathrm{~N}, \alpha \in] 0,1\left[\right.$ and $x, y \in \Gamma_{1}$ with $(x-y) / \Delta x \in{ }^{*} H$, then

$$
\left\|i_{x}-\dot{\tau}_{y}\right\|_{k} \leqq c_{10}|x-y|^{\alpha} v^{1-\alpha / 2},
$$

with

$$
c_{10}=c_{10}(k, \alpha)=4 c_{5} c_{6}(\alpha) k^{9 / 2}\left(1-2^{-(1-\alpha) / 2}\right)^{-1} .
$$

(ii) For all $x \in \dot{\Gamma}_{1}$ and $k \in 2 \mathrm{~N}$, we have

$$
\left\|\tau_{x}\right\|_{k} \leqq c_{10}(k, 0) v
$$

Like in Section 1, we can now use the nonstandard version of Kolmogorov's continuity theorem in order to conclude that the internal process $\tau_{\theta}:\left(T_{w} \times \Delta x^{*} H_{t}\right) \times \Omega \rightarrow{ }^{*} \mathrm{R}$ is $S$-continuous and has a projection $l_{l}:\left([0, w] \times \mathrm{R}^{2}\right) \times \Omega \rightarrow \mathrm{R}$, i.e. $l_{1}$ is a $C_{0}$-process such that

$$
{ }^{\circ} \dot{\tau}_{\boldsymbol{\theta}}(v ; x, \omega)=l_{l}\left({ }^{\circ} v ;{ }^{\circ} x, \omega\right) \quad\left(v \in T_{w}, x \in \Delta x^{*} H_{l}\right)
$$

holds for $P$-a.a. $\omega \in \Omega \quad(l=1, \ldots, r)$.

2.4. THEOREM. For every positive real $w$, the process $Y:[0, w]^{2} \times \Omega \rightarrow R$, $Y(s, t, \omega)=W(\mathrm{t}, \omega)-\mathrm{W}(\mathrm{s}, \omega)$, where $W$ is given by $(6)$, has a renormalized $C_{0^{-}}$ local time $\frac{1}{r} l,(w ;-,-): \mathrm{R}^{d} \times \Omega \rightarrow \mathrm{R}$ with respect to the time measure $\tilde{\Theta}_{l} \lambda \mid[0, w]^{2}$. Moreover, $l$, can be chosen jointly continuous and is given by (29).

Proof. Fix $\omega \in \Omega$ such that (6) and (29) hold. Pick $v \in T_{w}$ such that ${ }^{\circ} v=w$ and put $D=[0, w]^{2}, B=T_{v}^{2}$. Let $f: \mathrm{R}^{d} \rightarrow \mathrm{R}$ be an arbitrary continuous function with compact support. Then we have to check (12):

$$
\begin{aligned}
& \int_{D}\left[f(W(t, \omega)-W(s, \omega))-E f\left(W_{t}-W_{s}\right)\right] \tilde{\Theta}_{l}(s, t) d \lambda(s, t) \\
& ={ }_{(s, t) \in B}(\Delta t)^{2} \Theta_{l}(s, t)\left[{ }^{*} f(\beta(t, \omega)-\beta(s, \omega))-\underline{E}^{*} f\left(\beta_{t}-\beta_{s}\right)\right],
\end{aligned}
$$

by the same steps as in the proof of Theorem 1.12, using in addition the $S$-integrability of the function $(s, t ; \omega) \mapsto{ }^{*} f(\beta(t, \omega)-\beta(s, \omega))$ with respect to $(\Theta, \underline{\lambda} \mid B) \otimes \underline{P}$

$$
\begin{aligned}
& ={ }^{\circ} \sum_{x \in \Delta x^{*} H_{l}}(\Delta x)^{2} \dot{\tau}_{\boldsymbol{\theta}_{l}}(v, x ; \omega)^{*} f(x), \text { by the definition of } \dot{\tau}(\text { see }(25)) \\
& =\frac{1}{r} \int l_{l}\left({ }^{\circ} v ; x, \omega\right) f(x) d x, \text { by (29) as in the proof of Theorem 1.12. }
\end{aligned}
$$

2.5. Corollary. (i) If $\tilde{\Theta}_{1}=\ldots=\tilde{\Theta}_{r}=: \frac{1}{r} \theta$, then $\tau_{\theta}$ is $S$-continuous with 
standard part $l=l_{1}=\ldots=l_{r}$, which is the renormalized local time of $Y$ with respect to the time measure $\theta \lambda \mid D$.

(ii) Theorem 2.4 implicitly contains an invariance principle which can be stated in analogy to Corollary 1.13 (ii).

\section{Nonstandard Construction of Varadhan's Polymer Measure.}

In this section, we shall not only consider the specific realization (7) of the $\delta$-function, but an arbitrary internal $\Phi: \Gamma \rightarrow{ }^{*} \mathrm{R}_{+}$such that

$$
\sum_{x \in \Gamma} \Phi(x)(\Delta x)^{2} \leqq 1
$$

Let $L(\Phi)$ be the Loeb measure on $\Gamma$, induced by the internal measure on $\Gamma$ giving each $x \in \Gamma$ the weight $\Phi(x)(\Delta x)^{2}$. Then $m:=L(\Phi) \circ$ st $^{-1}$ is a measure on $\mathbf{R}^{2}$ with $m\left(\mathbf{R}^{2}\right) \leqq 1$. Every such measure $m$ can be represented in this way by a suitable internal $\Phi$. In general, $m$ is not absolutely continuous with respect to the Lebesgue measure $d x$ on $\mathrm{R}^{2}$. Nevertheless, we heuristically write $\tilde{\Phi}:=d m / d x$. So, if

$\Phi(x)=\left\{\begin{array}{ll}(\Delta x)^{-2}, & \text { if } x=0 \\ 0 & , \text { otherwise }\end{array} \quad(x \in \Gamma)\right.$,

then $\tilde{\Phi}$ is the $\delta$-function. The functional $J$ in the definition of the polymer measure (see (1), (2)) will then be represented by the internal random variable

$$
\Phi \tau: \Omega \rightarrow{ }^{*} \mathrm{R}_{+}, \omega \mapsto \sum_{x \in \Gamma} \tau_{\boldsymbol{\theta}}(v ; x, \omega) \Phi(x)(\Delta x)^{2},
$$

where $v \in \mathrm{ns}\left({ }^{*} \mathrm{R}_{+}\right)$and $\Theta: T \times T \rightarrow{ }^{*}[0,1]$ are fixed.

Note that by $(25)$ we have

$$
\Phi \tau(\omega)=\sum\left[(\Delta t)^{2} \Theta(s, t) \Phi(\beta(t, \omega)-\beta(s, \omega)) \mid s, t \in T_{v}\right]
$$

for all $\omega \in \Omega$. Fix $\underline{g} \in \mathrm{ns}\left({ }^{*} \mathrm{R}_{+}\right)$and define the internal polymer measure $v$ on $(\Omega, \underline{A})$ by giving it the $\underline{P}$-density

$$
\frac{d v}{d \underline{P}}(\omega)=\frac{1}{\underline{Z}} \exp [-\underline{g} \Phi \tau(\omega)] \quad(\omega \in \Omega),
$$

where $\underline{Z}=\underline{E}(\exp [-g \Phi \tau])$ is the normalization contant. Note that

$$
\frac{d v}{d \underline{P}}(\omega)=(1 / \underline{Z}) \exp [-g \Phi \dot{\tau}(\omega)] \quad(\omega \in \Omega),
$$

where $\underline{Z}=\underline{E}(\exp [-g \Phi \dot{\tau}])$ is another normalization constant and $\Phi \dot{\tau}$ is defined in analogy to (31). Our goal is to show that the partially defined measurable map 
$\bar{W}=$ st $\circ \beta:(\Omega, \underline{A}) \rightarrow C\left([0, w], \mathrm{R}^{2}\right)$ (where $\beta(\omega)=\beta(\cdot, \omega)$ and st is coming from the sup-norm) turns the Loeb measure $L(v)$ into Varadhan's polymer measure $v$, characterized by $w={ }^{\circ} v, g={ }^{\circ} \underline{g}, \tilde{\Phi}$, and $\tilde{\Theta}$. The main difficulty is to show that $\underline{Z}$ is finite. Then we can make use of the results in Section 2. We shall prove the finiteness of $\underline{Z}$ by a nonstandard version of Nelson's trick (see [12], [23]). To this end, we work with the Westwater domains given by Notation 2.1 and define $\Phi \tau_{\xi}$, $\Phi \sigma_{\xi}, \Phi \rho_{\xi}$ in analogy to (31). Our application of Nelson's trick is based on the following estimates:

3.1. Lemma. For all $\xi \in\{0,1, \ldots \kappa\}$, we have

(i) $\underline{\operatorname{Var}}\left(\Phi \sigma_{\xi}\right) \leqq c_{11} v^{2} 2^{-\xi}$ with $c_{11}=\left(1-2^{-1 / 2}\right)^{-2} 2^{13}\left(c_{5} c_{6}\right)^{2}$.

(ii) $\underline{E}\left(\Phi \tau_{\xi}\right) \leqq c_{12} v \xi$ with $c_{12}=2 c_{5} c_{6}$.

ProOF. (i) $\left[\underline{\operatorname{Var}}\left(\Phi \sigma_{\xi}\right)\right]^{1 / 2} \leqq \sum_{\zeta=\xi}^{\kappa}\left\|\Phi \dot{\rho}_{\zeta}\right\|_{2}$, by notation 2.1 ,

$$
\begin{aligned}
& \leqq \sum_{\zeta=\xi}^{\kappa} \sup _{x \in \Gamma}\left\|\rho_{\zeta}(x,-)\right\|_{2} \text {, by }(31),(30) ; \\
& \leqq \sum_{\zeta=\xi}^{\kappa}\left(4 c_{5} c_{6} 2^{9 / 2} v 2^{-\zeta / 2}\right), \text { by }(28)
\end{aligned}
$$

(notice that $\rho_{\zeta}(x, \omega)=0$ for all $\left.x \in \Gamma \backslash \Gamma_{1}\right)$;

$$
\leqq\left(1-2^{-1 / 2}\right) 2^{13 / 2} c_{5} c_{6} v 2^{-\xi / 2} .
$$

(ii) $\underline{E}\left(\Phi \tau_{\xi}\right)=\sum_{\zeta=0}^{\xi-1} \sum_{\eta=0}^{2^{\zeta}-1} \sum_{j=1}^{2} E\left(\Phi \tau_{\theta}^{v}\left[\Lambda^{\zeta}(n, j)\right]\right)$, by Notation 2.1 .

$$
\begin{aligned}
& \leqq \sum_{\zeta=0}^{\xi-1} \sum_{\eta=0}^{2^{\zeta}-1} 2 \sup _{x \in \Gamma}\left\|\tau_{\theta}^{v}\left[\Lambda_{\zeta}(\eta, 2)\right](x,-)\right\|_{1} \text {, by }(31) ; \\
& \leqq \sum_{\zeta=0}^{\xi-1} \sum_{\eta=0}^{2^{\zeta}-1} 2 c_{5} c_{6} v 2^{-\zeta}, \text { by }(27) ; \\
& \leqq 2 c_{5} c_{6} v \xi
\end{aligned}
$$

3.2. PROPOSITION.

$$
\underline{Z}:=\underline{E}(\exp [-\underline{g} \Phi \dot{\tau}]) \text { is finite }
$$

for all finite $\underline{g}, v \in * R_{+}$.

Proof. (i) Let us first consider the case that $v \leqq 1$ and $v \cdot g \leqq c_{13}:=(\log 2) /\left(2 c_{12}\right)$. Furthermore, we may assume that $\underline{g}>0$ and $v \geqq \Delta t$. Then for all $\xi \in\{1,2, \ldots, \kappa\}$, we have: 


$$
\begin{aligned}
& \underline{P}\left(\Phi \dot{\tau} \leqq-2 c_{12} v \xi\right) \\
& \leqq \underline{P}\left(\Phi \dot{\sigma}_{\xi} \leqq-2 c_{12} v \xi+\underline{E}\left(\Phi \tau_{\xi}\right)\right), \text { by } \tau=\sigma_{\xi}+\tau_{\zeta} \text { and } \tau_{\xi} \geqq 0 ; \\
& \leqq \underline{P}\left(\Phi \dot{\sigma}_{\xi} \leqq-c_{12} v \xi\right), \text { by Lemma } 3.1 \text { (ii); } \\
& \leqq\left(c_{12} v\right)^{-2} \xi^{-2} \underline{\operatorname{Var}}\left(\Phi \sigma_{\xi}\right), \text { by Chebyshev's inequality; } \\
& \leqq c_{11} c_{12}^{-2} 2^{-\xi} \xi^{-2}, \text { by Lemma } 3.1 \text { (i). }
\end{aligned}
$$

On the other hand, by integral transformation we get

$$
\begin{aligned}
& \underline{E}[\exp (-\underline{g} \Phi \dot{\tau})]=\int_{0}^{\infty} \underline{P}(\exp (-\underline{g} \Phi \dot{\tau}) \geqq t)^{*} d t \\
& \leqq 1+\int_{0}^{\infty} \underline{P}(\Phi \dot{\tau} \leqq-s) e^{\underline{g} s *} d s, \text { by the substitution } s=(\log t) / \underline{g} \\
& \leqq 1+\int_{0}^{2 v c_{12}} e^{\underline{g} t *} d t+\sum_{\xi=1}^{\kappa-1} \underline{P}\left(\Phi \dot{\tau} \leqq-2 v c_{12} \xi\right) 2 v c_{12} \exp \left[2 \underline{g v} c_{12}(\xi+1)\right] \\
& \text { since } \Phi \dot{\tau} \geqq-\underline{E}\left(\Phi \tau_{\kappa}\right) \geqq-v c_{12} \kappa \text { by Lemma } 3.1(\mathrm{ii}) ; \\
& \leqq 1+2 v c_{12} \exp \left[2 v \underline{g} c_{12}\right]+2 v\left(c_{11} / c_{12}\right) \exp \left[2 v \underline{g} c_{12}\right] \cdot \\
& \sum_{\xi=1}^{\kappa-1} 2^{-\xi} \xi^{-2} \exp \left[2 v \underline{g} c_{12} \xi\right], \text { by }(34) ; \\
& \leqq 1+2 c_{12} \exp \left[2 c_{12} c_{13}\right]+2\left(c_{11} / c_{12}\right) \exp \left[2 c_{12} c_{13}\right] . \\
& \sum_{\xi=1}^{\kappa-1} \xi^{-2} \exp \left[-\xi\left(\log 2-2 c_{12} c_{13}\right)\right], \text { by } v \cdot \underline{g} \leqq c_{13} \text { and } v \leqq 1 \\
& \leqq 1+4 c_{12}+4\left(c_{11} / c_{12}\right) \sum_{\xi=1}^{\kappa-1} \xi^{-2}, \text { by } c_{13}=(\log 2) /\left(2 c_{12}\right) ; \\
& \leqq 1+4 c_{12}+8\left(c_{11} / c_{12}\right)=: c_{14} .
\end{aligned}
$$

(ii) In the case not covered by (i), $v$ is not infinitesimal, in particular $\kappa \in * N \backslash N$ (cf. (26)). Choose $n \in \mathrm{N}$ such that $2^{n}>2 v\left(1 \vee 2 \underline{g} / c_{13}\right)$. Hölder's inequality gives

$$
\underline{E}[\exp (-\underline{g} \Phi \dot{\tau})] \leqq\left(\underline{E}\left[\exp \left(-2 \underline{g} \Phi \dot{\tau}_{n}\right)\right]\right)^{1 / 2}\left(\underline{E}\left[\exp \left(-2 \underline{g} \Phi \dot{\sigma}_{n}\right)\right]\right)^{1 / 2},
$$

Moreover,

$$
\left(\underline{E}\left[\exp \left(-2 \underline{g} \Phi \dot{\tau}_{n}\right)\right]\right)^{1 / 2} \leqq \exp \left(\underline{g} \underline{E}\left[\Phi \tau_{n}\right]\right) \leqq \exp \left(c_{12} v \underline{g} n\right),
$$

by Lemma 3.1 (ii). Thus it remains to show the finiteness of

$$
E\left[\exp \left(-2 \underline{g} \Phi \dot{\sigma}_{n}\right)\right]=\sum_{\eta=0}^{2^{n}-1} \underline{E}\left[\exp \left(-2 \underline{g} \Phi \dot{\tau}_{\theta}^{v}\left[\Delta_{n}(\eta)\right]\right)\right]
$$


Fix $\eta \in\left\{0,1, \ldots, 2^{n}-1\right\}$. Put $\bar{v}:=2^{\kappa-n} \Delta t$. Note that

$$
\bar{v} \leqq 2^{-(n-1)} v \leqq 1 \text { and } 2 \bar{v} \underline{g} \leqq 2^{-(n-2)} v \underline{g} \leqq c_{13}
$$

by the choice of $n$. Define the internal $\bar{\Theta}: T^{2} \rightarrow^{*}[0,1]$ by $\bar{\Theta}(s, t)=\Theta\left(s+\eta 2^{\kappa-n} \Delta t, t+\eta 2^{k-n} \Delta t\right) \cdot \chi r_{v}^{2}(s, t)$. Then we have

$$
\begin{aligned}
E\left[\exp \left(-2 \underline{g} \Phi \dot{\tau}_{\boldsymbol{\theta}}^{v}\left[\Delta_{n}(\eta)\right]\right)\right] & =\underline{E}\left[\exp \left(-2 \underline{g} \Phi \dot{\tau}_{\bar{\theta}}(\bar{v} ;-,-)\right)\right] \\
& \left.\leqq c_{14}, \text { by (i) because of } 36\right) .
\end{aligned}
$$

Since $\eta \in\left\{0,1, \ldots, 2^{n}-1\right\}$ was arbitrary, (35) implies

$$
\underline{E}\left[\exp \left(-2 \underline{g} \Phi \dot{\sigma}_{n}\right)\right] \leqq\left(c_{14}\right)^{2^{n}}<\infty .
$$

Proposition 3.2 implies that the internal density $d v / d \underline{P}$, given by (32) or (33), belongs to the class $\mathrm{SL}^{P}$ (defined as in Anderson [2]) for all real $p>1$. Let us now determine its projection. For simplicity, let us assume $\tilde{\Theta}_{1}=\ldots=\widetilde{\Theta}_{r}=\frac{1}{r} \theta$. The extension to the general case is obvious. Moreover, let $w={ }^{\circ} v>0, g={ }^{\circ} g \in \mathbf{R}_{+}$.

If $\omega \in \Omega$ is such that (29) holds, then

$$
\begin{aligned}
{ }^{\circ} \Phi \dot{\tau}(\omega) & ={ }_{x \in \Gamma} \dot{\tau}_{\boldsymbol{\theta}}(v ; x, \omega) \Phi(x)(\Delta x)^{d}, \text { by }(31) ; \\
& =\int_{\mathrm{ns}(\Gamma)}{ }^{\circ} \dot{\tau}_{\boldsymbol{\theta}}(v ; x, \omega) L(\Phi)(d x),
\end{aligned}
$$

since $\dot{\tau}_{\theta}(v ;-, \omega)$ is $S$-integrable with respect to $L(\Phi)$;

$$
\begin{aligned}
& =\int_{\mathrm{ns}(\Gamma)}\left[\left({ }^{\circ} v ;{ }^{\circ} x, \omega\right) L(\Phi)(d x), \text { by }(29) ;\right. \\
& =\int i(w ; x, \omega) m(d x)=: m l \text {, by } L(\Phi) \circ \mathrm{st}^{-1}=m ; \\
& =\int_{0}^{w} d s \int_{0}^{w} d t \theta(s, t) \frac{d m}{d x}[W(t, \omega)-W(s, \omega)]-E(\ldots), \text { by }(13) .
\end{aligned}
$$

Therefore

$$
{ }^{\circ} \exp [-\underline{g} \Phi \dot{\tau}]=\exp [-g m i[] \underline{P} \text {-a.s. }
$$

Then with proposition 3.2 we infer that

$$
\infty>{ }^{\circ} \underline{Z}={ }^{\circ} \underline{E}[\exp (-\underline{g} \Phi \dot{\tau})]=E[\exp (-g m l)]=: \dot{Z} .
$$


Thus we may define a 'polymer' measure $v$ on $C\left([0, w], \mathrm{R}^{2}\right)$ by the Wienerdensity

$$
\frac{d v}{d \mu} \circ \bar{W}=(1 / Z) \exp [-g m \dot{l}] \underline{P} \text {-a.s. }
$$

Notice that $v$ is well defined by (39), because $\mu=P \circ \bar{W}^{-1}$ and $l$ is measurable with respect to the $P$-completed $\sigma$-algebra generated by $\bar{W}$. From (37) and (38) we can conclude that $d v / d \underline{P}=\exp (-\underline{g} \Phi \dot{\tau}) / \underline{Z}$ is a $S$-integrable lifting of $\exp [-g m i] / Z$ and hence

$$
\frac{d L(v)}{d L(\underline{P})}=(1 / Z) \exp [-g m i ́]=\frac{d v}{d \mu} \circ \bar{W} \quad P \text {-a.s. }
$$

Since $\mu=L(\underline{P}) \circ \bar{W}^{-1}$, we have obtained:

\subsection{MAIN THEOREM. $v=L(v) \circ \bar{W}^{-1}$.}

Routine applications of the permanence principle show that Theorem 3.3 implies the following results, which are formulated purely in standard notation:

3.4. Corollary. (i) The operator $(g, \theta, m) \mapsto v$ is continuous in the sense that we have $v_{n} \underset{n \rightarrow \infty}{\stackrel{\text { weakly }}{\longrightarrow}} v$, if $v_{n}$ is constructed with $g_{n}, \theta_{n}, m_{n}$ such that $g_{n} \underset{n \rightarrow \infty}{\longrightarrow}$,

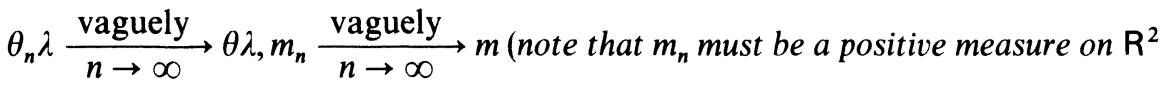
with $\left.m_{n}\left(\mathbf{R}^{2}\right) \leqq 1\right)$. In particular, we may choose $m_{n}(B)=\int \chi_{B}(x) f_{n}(x) d x$, where $f_{n}: R^{2} \rightarrow R_{+}$is continuous and bounded. Then we have

$$
\frac{d v_{n}}{d \mu}(\omega)=\frac{1}{Z_{n}} \exp \left[-g_{n} \int_{0}^{w} d s \int_{0}^{w} d t \theta_{n}(s, t) f_{n}(\omega(t)-\omega(s))\right]
$$

for $\mu$-a.a. $\omega \in C\left([0, w], \mathrm{R}^{2}\right)$.

Since the right hand side of $(40)$ is well-defined, this gives an alternative possibility, to define the polymer measure $v$ for arbitrary $m$, i.e. to give a precise meaning to the heuristic expression

$$
\frac{d v}{d \mu}(\omega)=\frac{1}{Z} \exp \left[-g \int_{0}^{w} d s \int_{0}^{w} d t \theta(s, t) \frac{d m}{d x}(\omega(t)-\omega(s))\right] .
$$

In particular, we have recovered Varadhan's results (see [23]).

(ii) Moreover, we obtain an invariance principle for the polymer measure $v$, i.e. $v$ can be approximated by elements of a certain class of self-repellent random walks. Assume the same setting as in Corollary 1.13 (ii) and let $d=2$. Furthermore, for each $n \in \mathrm{N}$, let $\Gamma_{n}=\left\{\left(k_{1} \Delta x_{n}, k_{2} \Delta x_{n}\right): k_{1}, k_{2} \in \mathrm{Z}\right\}, G_{n} \in \mathrm{R}_{+}$, and $m_{n}$ an arbitrary positive measure on $\Gamma_{n}$ with $m_{n}\left(\Gamma_{n}\right) \leqq 1$. For each $n \in \mathrm{N}$ define the 
probability measure $v_{n}$ on $\left(\Omega_{n}, A_{n}\right)$ by giving it the $P_{n}$-density

$$
\left(d v_{n} / d P_{n}\right)(\omega)=\left(1 / Z_{n}\right) \prod\left[\exp \left(G_{n} \theta_{n}(s, t) m_{n}\left\{\beta_{n}(t, \omega)-\beta_{n}(s, \omega)\right\}\right) \mid s, t \in T_{w}\right]
$$

for $P_{n}$-a.a. $\omega \in \Omega_{n}$. Suppose that

(0) $\lim \Delta t_{n}=0$;

$n \rightarrow \infty$

(1) $\lim \left(G_{n} / \Delta t_{n}\right)=g$;

$n \rightarrow \infty$

(2) $\theta_{n, l} \lambda_{n} \underset{n \rightarrow \infty}{\stackrel{\text { vaguely }}{\longrightarrow}} \frac{1}{r} \theta \lambda \quad(l=1, \ldots, r)$;

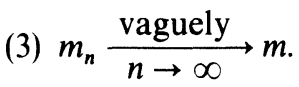

Then we have $\left(\beta_{n}, v_{n}\right) \underset{n \rightarrow \infty}{\stackrel{w}{\longrightarrow}} v$, i.e.

$$
v_{n} \circ\left(I_{n} \circ \bar{\beta}_{n}\right)^{-1} \underset{n \rightarrow \infty}{\stackrel{\text { weakly }}{\longrightarrow}} v
$$

for every reasonable sequence $\left(I_{n}\right)_{n \in \mathrm{N}}$ of interpolations $I_{n}: F_{0}\left(T_{n, w}, \mathrm{R}^{2}\right) \rightarrow C\left([O, w], \mathrm{R}^{2}\right)$.

\section{REFERENCES}

1. S. Albeverio, J.-E. Fenstad, R. Høegh-Krohn and T. Lindstrøm, Nonstandard Methods in Stochastic Analysis and Mathematical Physics, , Academic Press, New York, 1986.

2. R. M. Anderson, A non-standard representation for Brownian motion and Itô integration, Israel J. Math. 25 (1976), 15-46.

3. A. N. Borodin, On the asymptotic behavior of local times of recurrent random walks with finite variance (English translation), Theory Prob. Appl. 26 (1981), 758-772.

4. N. J. Cutland, Nonstandard measure theory and its applications, Bull. London Math. Soc. 15 (1983), 529-589.

5. C. Domb, Self-avoiding walk on lattices in Stochastic Processes in Chemical Physics (ed. K. E. Schuler), 229-260, Wiley, New York, 1969.

6. C. Domb, G. S. Joyce, Cluster expansion for a polymer chain, J. Phys. C 5 (1975), 956-976.

7. E. B. Dynkin, Random fields associated with multiple points of the Brownian motion, J. Funct. Anal. 62 (1985), 397-434.

8. S. F. Edwards, The statistical mechanics of polymers with excluded volume, Pro. Phys. Soc. 85 (1965), 613-624.

9. K. F. Freed, Polymers as self-avoiding walks, Ann. Probab. 9 (1981), 537-556.

10. D. Geman, J. Horowitz, J. Rosen, A local time analysis of intersections of Brownian paths in the plane, Ann. Probab. 12 (1984), 86-107.

11. J. F. Le Gall, Sur le temps local d'intersection du mouvement Brownien plan, et la méthode de renormalisation de Varadhan in Séminaire de Probabilités XIX, Lecture Notes in Mathematics 1123, 314-331. Springer, Berlin, 1985. 
12. E. Nelson, $A$ quartic interaction in two dimensions in Mathematical Theory of Elementary Particles, (eds. R. Goodman, I. Segal), 69-74, Cambridge, Mass., 1966.

13. E. Perkins, Weak invariance principles for local time, Z. Wahrscheinlichkeitstheorie verw. Geb. 60 (1982), 437-451.

14. J. Rosen, A local time approach to the self-intersections of Brownian paths in space, Comm. Math. Phys. 88 (1983), 327-338.

15. J. Rosen, Tanaka's formula and renormalization for intersections of planar Brownian motions, Ann. Probab. 14 (1986), 1245-1251.

16. J. Rosen, A renormalized local time for multiple intersections of planar Brownian motion, Séminaire de Probabilités XX, Lecture Notes in Mathematics 1204, 515-531, Springer, Berlin, 1987.

17. J. Rosen, Joint continuity of the intersection local time of Markov processes, Ann. Probab. 15 (1987), 659-675.

18. N. R. Sieh, The self-intersections of diffusion paths, Preprint, 1985.

19. F. Spitzer, Principles of Random Walk, 2nd ed. Springer, Berlin, 1976.

20. A. Stoll, Self-Repellent Random Walks and Polymer Measures in Two Dimensions, Ph. D. Thesis, Ruhr-Universität Bochum, 1985.

21. A. Stoll, A nonstandard construction of Lévy Brownian motion, Probab. Th. Rel. Fields 71 (1986), 321-334.

22. K. D. Stroyan and J. M. Bayod, Foundations of Infinitesimal Stochastic Analysis, North-Holland, Amsterdam, 1986.

23. S. R. S. Varadhan, Appendix to 'Euclidean quantum field theory' by K. Symanzik, in Local Quantum Theory (ed. R. Jost), 219-226. Academic Press, New York, 1969.

24. S. Weinryh Image par une mesure de $\mathrm{R}^{3}$ de lintersection de deux saucisses de Wiener indépendantes; lemps locaux de'intersection relatifs à cette mesure, Preprint, 1985.

25. J. Westwater, On Edwards' model for long polymer chains, Comm. Math. Phys. 72(1980), 131-174.

26. J. Westwater, On Edwards' model for polymer chains III; Borel summability, Comm. Math. Phys. 84 (1982), 459-470.

27. M. Yor, Compléments aux formules de Tanaka-Rosen, Seminaire de Probabilités XIX, Lecture Notes in Mathematics 1123, 332-349, Springer, Berlin, 1985.

28. M. Yor, Sur la représentation comme intégrales stochastiques des temps d'occupation du mouvement Brownien dans $\mathbf{R}^{d}$, Séminaire de Probabilités XX, Lecture Notes in Mathematics 1204, 543-552, Springer, Berlin, 1987.

ABTEILUNG FÜR MATHEMATIK

RUHR-UNIVERSITÄT

4630 BOCHUM I

WEST GERMANY 